\title{
A COMPUTER SIMULATION OF THE CREATION OF A TRANSIENT, HIGH-DENSITY PLASMA BY CONVERGENT NEUTRAL BEAMS
}

\author{
C. J. Eggens (Hartman)
}

June 1, 1976

Prepared for U.S. Energy Research \& Development Administration under contract No. W-7405-Eng-48

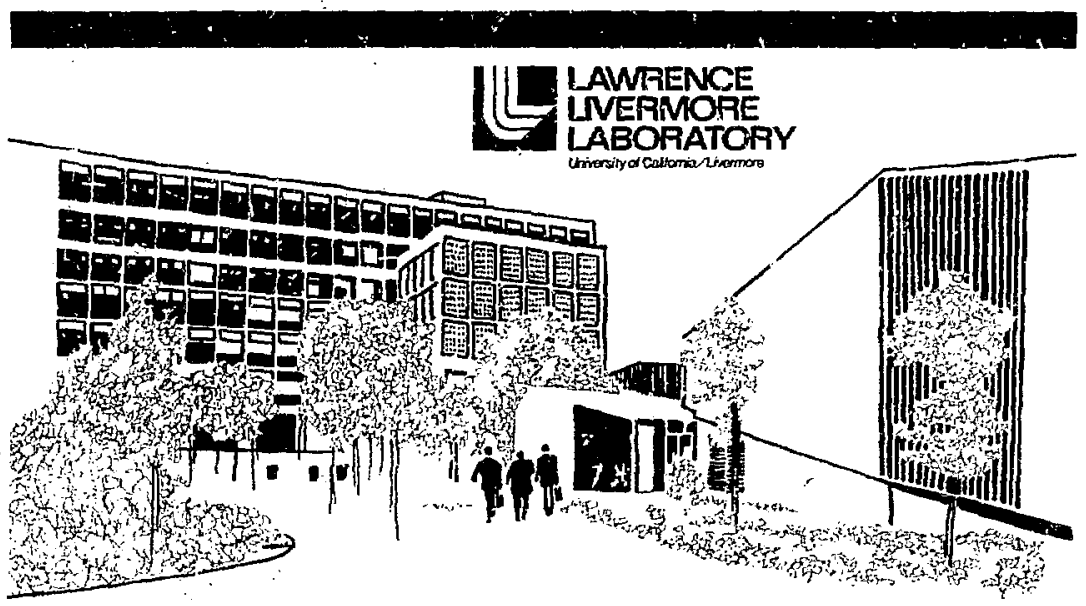





\title{
L4 \\ LAWRENCE LNERMORE LABORATORY \\ University of Cartamia Livemore, Cahiomia 94550
}

\section{LCRL-52055 \\ A COMPUTER SIMULATION OF THE CREATION OF A TRANSIENT, HIGH-DENSITY PLASMA BY CONVERGENT NEUTRAL BEAMS}

\author{
C. J. Eggens (Hartwan) \\ MS. date: June 1,1976
}

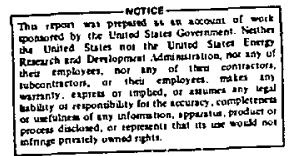




\section{Contents}

Abstract . . . . . . . . , , . . . . . . . . . . . . 1

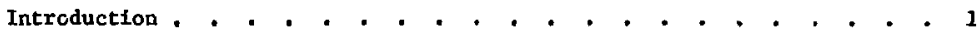

Neutral Beams , . . . . . . . , , . , , . . . . . . . 2

The Convergencer-Bunching Technfque . . . . . . . . . . . 3

Nithematical Representacion of Physics of

Convergent Neutral Beams . . . . . , . . . . . 5

Spatial Disergence Vue to Ion Optics . . . . . . . . . 5

Focusing Uncertainty Along Axis , , , , , . . . . 6

Fcasibility of Method + , . , , . . , , . , . . , 8

Numerical Solution of Model . . . . . . . , . . . , . . . 10

General Approach . . . . . . . . . . . . . . . . 10

Distribution Function. . . . . . . . . . . . . . . . 11

Number Denstey . . . . . . . . . . . . . . . . . . 13

Spherical Geometry. . . . . . , , . . . . . . 14

Cylindrical Geometry . . . . . . . . . . . . . 22

Ionizatior Probabilities . , . . . . . . . . . . . . . 25

Spherical Geometry , . . . . . . . . . . . . . 27

Cylindrica1 Geometry . . . . . . . . . . . . . . 29

Computer Code . . . . . . . . . . . . . . . . . . 31

Discussior of Plasma Creation . . . . . . . . . . . . . . . 33

Summary . . . . . . . . . . . . . . . . . . . . . . 39

Acknowledgments , . . . . . . . . . . . . . . . . . . , 41

Appendix A. Characteristics of Neutxal Beams , . . . . . . . . . 42

Appendix B, Solution to Vlasov Equation Fithin an

Ion Source . . . . . . . . . . . . 45

Appendix C. Llouville's Theoren . . . . . . . . . . . . . . 48

Appendix D, Approximate Representation of

Distribution Function . . . . . . . . 49

Appendix E, Error Determination for Trapezoidal Integration . . . . 51

Appendix F. Error in the Probability Calculation . . . . . . . . 53

Appendix G. Derlvation of Number Densities from the

Continuity Equation for Times Prior to

Convergence............. 54

References , . . . . . . . . . . . . . . . . 56 


\section{A COMPUTER SIMULATION OF THE CREATION OF A TRANSIENT, HIGH-DENSITY PLASMA BY CONVERGENT NEUTRAL BEAMS}

\section{Abstract}

A computer sfmulation of the creation of a transient, high-density plasma by convergent neutral beams establishes a formalism through which accurate predictions of the geometrical convergence and the temporal bunching of neutral beams can be made as they proceed. Numerícal studies of convergent neutral beams include calculation of number densities and the degree of lonization of the neutral distribution as the beams approach convergence. The calculations encompass both spherical and cylindrical geometries. The distribution functions obtalned from a mathematical model are integrated numerically to obtain particle densities. Values of the lonization rate parameter $\overline{O V}_{\mathrm{NN}}$ are simflarly obtained using numerical fits to the neutral-neutral lonization cross-section data. Integration of the resulting lonization rate provides a first-order estimate of the Ion density $n_{I}$ as a function of space and time. $A$ better calculation of $\mathrm{t}_{I}$ is then obtained by adding the second-order effects nf neutral-ion collisione. It is thereby possible to determine whether the distribution will be significantly lonized before th has completely converged. Theory had suggested that temporal and spatial convergence of neutral beams would create a maximum density at the target center that would correspond to an enhancenent of the Initfal beam Jensitles by many orders of magaltude. Results of calculations confirm that the suggested techrique could have Important application in fusion power research.

\section{Introduction}

Neutral-beam injection is emerging as a powerful tool in fuslon research. P) eliminary calculations showed that, bscause of recent advances in the technology of high-current, pulsed neutral beams, it wight be posstble to create a translent, high-density plasma by combint.ng focused, spatial convergence wth the temporal bunching achieved by velocity modulation. 1,2 However, a more rigorous proof was required. 


\title{
A COMPUTER SIMULATION OF THE CREATION OF A TRANSIENT, HIGH-DENSITY PLASMA BY CONVERGENT NEUTRAL BEAMS
}

\begin{abstract}
A conputer stmulation of the creation of a transient, high-denstty plasma by convergent neutral beams establishes a formailsm through which accurate predictions of the geometrical convergence and the temporal bunching of neutral beams can be made as they proceed. Numerical studies of convergent neutral beams include calculation of number densittes and the degree of ionization of the neutral distribution as the beams approach convergence. The calculations encompass both spherical and cylindrieal gectetrizs. The diatribution functions obtained from a mathematical model are Integrated nwerically to obtain particle densities. Values of the lonlation rate parameter $\overline{\sigma v_{N N}}$ are similarly optained using numerical fits to the neutral-neutral lonization cross-section aate. Integration of the resulting ionization rate provides a first-order estimate of the Ion denstey $n_{I}$ as a function of space and time. A better calculation of $n_{I}$ is then obtained by adding the second-order effects of neutral-ion collistons. It is thereby posstble to deternine whather the distribution w111 be signiflcantly lonized before th has completely converged. Theory had suggested that temporal and spatlal convergence of neutral beans wcild create a maximum density at the target center that would correspond to an enhancement of the Initlal beam densities by many orders of magnitude. Results of calculations confirm tiat the suggested technique could have important application in fusion power research.
\end{abstract}

\section{Introduction}

Neutral-beam injection is energing as a powerful tool in fusion research. Preliminary calculations showed that, b scause of recent advances in the technology of high-current, pulsed neutral beams, it might be possible to create a transient, high-density plasma by comblning focused, spatial convergense with the temporal bunching achleved by velocity modulation. 1,2 However, a more rigorous proof was required. 
This report descritos a computer code written to establish a formalism through which accurate predictions of the geometrical convergence and tine temporal bunching of neutral beams could be made as they proceed. ${ }^{3}$ Approximate calculations ${ }^{4}$ are extended and verified in detailed calculations. In the latter, an intial distribution function ac the source is derived fron a solution to the Vlasov equation through the electrode structure of the fon source. Liouville's theorem is then applied to follow the evolution oi this distribution function. The code evaluates the distribution function at any pusition and time within the beam region. Additfonally, it calculates the neutral aton density, the relative velocity, the rate parameters $\overline{\sigma v}_{\mathrm{NN}}, \overline{\sigma v}_{\mathrm{NI}}$, the probabilities of ionization, and the ion density. The results of these calculations confint that the convergence-bunching technique could have important application in fuston power research.

The report concludes with predictions of the subsequent history of the newly created plasma. Appendices provide supplementary Luformation and derivations.

\section{Neutral Beams}

Achlevement rf intense, highly collimated neutral beams is cruclal to the success of the convergence-bunching technique. Only recently have appropriate sources been avallable, and much research is still needed, $5,6,7$

Ion sources and neutral beams have had a long and progressive history of varied applications and requirements. Some of the considerations of intense highly collimated neutral beams discussed here were a result of much effort. for other applications and at other energies.

Neutral beams are produced from lons and have an intrinsic divergence due to physical and mechanical 1imitations of the plasma sources. The ions must be formed, extracted, accelerated, and charge neutralized. Each $\%$ ress must be optimlzed to limit, as much as posslble, the bean divergenc :aliy mechanism that would disperse the intense ion beam before it is converted into neutral particles must be avoided. Appendix A discusses the fundamental processes that limit the important beam parameters of divergence and intensity.

Energetic neutral atomic and molecular beams were first suggested in the $1950^{\prime} \mathrm{s}$ as a means of filling magnetic bottles with fusion-temperature plasmas. The injection of neutral (and ton) beams into mirror machines to create, sustain, and heat plasmas evolved from 1 complicated and continuing technology. 
This application is a major component of the program to develof the wagnetic mirrur concept for fusion and was a major impetus for the development of highintensity sources. ${ }^{8}$

Recent technology has led to the extension of beams to higher power and better focusinga Large-volume, highly uniform plasmas ${ }^{7}$ are being used, and che beam-focusing optics are being alded by sophisticated computer simuletion. 5

The Lawrence Berkeley Laboratory (LBL) $50-A$ source ${ }^{6}$ is one or the most promising for Intense, pulsed neutral-beam appiications. Development of these beams has progressed to the point where alternate proposals for controlled thermonuclear fusion seem feasible. In particular, it is hoped that these beams could be relocity modulated so that the bunching technique proposed here could be applied.

THE CONVERGENCE-BUNCHISG TECHNIQUE

The proposed method for creating transient, high-density plasma ${ }^{2}$ is based on the tineory that the coicicidence of temporal and spatial convergence of the intense, low-divergence neutral beams would creace a maximum density at the target center that would correspond to an enhancement of the initial beam densities by many orders of magnitude. The beams would be radially focused on the surface of a sphere or cylinder and directed toward a center target site by the directed motion imparted to the particle currents by the curved surface. For example, as the center of a spherfcal containment vesse? is approached, particle density would increase froportionally to $1 / \mathbf{r}^{2}$. Figure 1 shows how an array of inwardly directed, pulsed neutral-beam sourcss located on a spherical surface would spatially converge.

In addition to spatial convergence, it is proposed to velocity modulate the beins so that all the celtral partlcles arrive at the targer site simultaneously. This technique, which utillzes the idea of a klystron, is shown schematically in ig. 2 .

The proposed energy (velocity) modulation of the neutral beam is

$$
\begin{aligned}
& W_{0}(t)=\frac{W_{I}}{\left(1-t / t_{0}\right)^{2}}, \\
& v_{0}(c)=\frac{v_{I}}{\left(1-t / t_{0}\right)},
\end{aligned}
$$




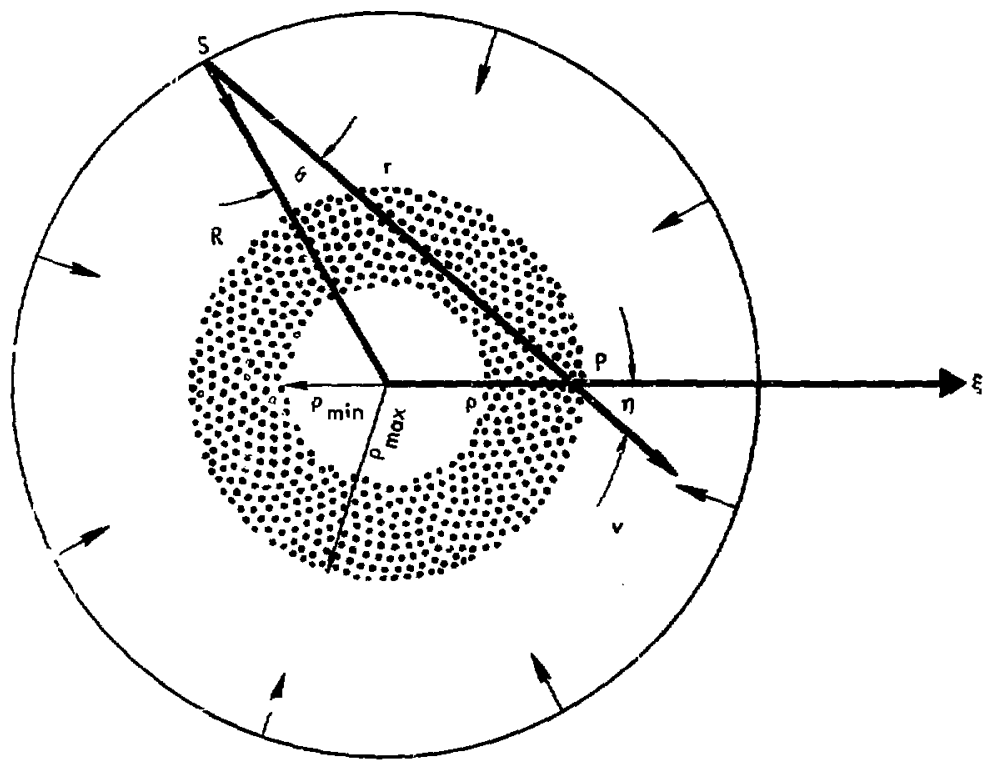

Fig. 1. Spatlal convergence of Incoming convergent neutral beams.

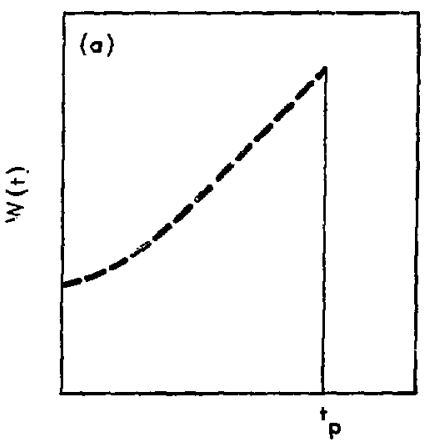

Time, +

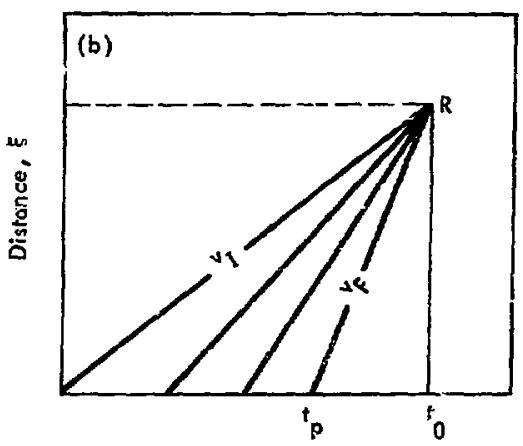

Time, $t$

F1g. 2. a) Temporal convergence through energy (velocity) modulation of neutral beams. b) Schematic showing neutrals with different velocttlea, emitted at different times, all reach same point $R$. 
At time $c=0$, neutral atoms are liunched with an intalal energy $w_{I}$ (and velocity $v_{I}$ ). With the progress of time, the beam energy is programmed up (by changing the acceleration potential in ti,e ion gun portion of the source) so that particles launched late in time would catch up wh the earlier ones. Because neutral beams are essentlally noninteractive, it may be possible to arrange an array of sources on a sphere or cylinder to creace high instantaneous power densities ac the colncidence of temporal and spatial convergence. At the target point, elther collisional self-ionization or lonization by impact on a target plasma would result in the conversion of the neutral partfcle cloud to a transient, bigh-density plasma.

Note that the required coilfiguration would be large $(=300 \mathrm{~cm})$; a1so, the sources now avallable wo:id be applicable only to a cylindrical concainment vessel. Further source development is necessary before the spherical geometries and velocity modulation ho;ed for in the proposed technique vould be posisible.

\section{MATHEMATICAL REPREGENTATION OF PINSICS OF CONVERGENT NEUTRAL BEATS}

Characteristic of the convergent neutral-beam system is the accuracy of the spatial and terporal focusing. The uncertainfles involved wth beam colIImarfun at the target site are represented as follows in variables pertinent to the rystem:

$\mathrm{J}_{0} \quad=$ current density at source.

$W_{I}\left(v_{I}\right)=$ initial energy (velocity) of neutrals at an emfssfon $t$ ine $t=0$.

$\mathrm{t}_{0}=$ characteristic flight time

$t_{p}=$ F'ise duration

$\stackrel{p}{\Delta W_{\perp}} \quad=$ angular beam divergence (predominately determined by ton themal energy).

$\Delta W_{\|} \quad=$ uncertafinty due to velocity modulation.

$\mathrm{R} \quad=$ radial distance between source and target center.

\section{Spatial Divergence Due to Ion Optics}

As discussed earlier, physical and mecharical liutrarions cause all beams to diverge. The degree of convergence that san be achleved is prinarily 11mited by the angular divergence of the neutral atom beam. This in turn is ultimately deternined by the themal energy $\Delta_{1}$ of the initial plasma saurce 
from which the ions were extracted. The angular divergence is perpendicular to the beam. The neutrals, then, arrive in the vicinity of the target sfte within an uncertainty given by

$$
\Delta S_{\perp}=R \sqrt{\frac{\Delta W_{1}}{W_{I}}} .
$$

As shown in Fig. 3(a), this relacionship is simply derived by applying the small-angle formula for the spread $\delta$ after the beam has traversed a distance $R$ froll the source, $S$. If there were no divergence, the beam would he radially directed inward along the axis $\xi$ to the center of the sphere, 0 . For straight-1ine trajectorles, the velocity vectors are in the same direction and are proportional to the position vector, so that

$$
\delta=\tan \delta=\frac{\Delta S_{1}}{R}=\frac{\Delta v_{\perp}}{v_{I}}=\sqrt{\frac{\Delta W_{1}}{W_{I}}} .
$$

\section{Focusing Uncertainty AZong Axis}

The ability to temporally bunch the neutral beam is also intrinsic to the proposed technique. If the pulse could be timed to collapse at the exact center of the sphere or cylinder, the target region would received a uniform energy density Input.

It is assumed that the neutral-beam sources provided can be energy (velocity) modulated without further degradation to the ion optics just described. If the angular divergence can be maintained independentiy of the accelerating potential, a longltudinal uncertainty corresponding to the spread In the distribution function as it emerges from the source is

$$
\Delta \mathbf{S}_{\|}=\frac{\mathrm{R} \Delta \mathrm{W}_{\|}}{2 \mathrm{~W}_{\mathrm{I}}}
$$

This is show schematically in Fig. 3(b).

The energy of the beam along the axis in this direction is given by the sum of the 1, Sal energy of the neutrals $W_{I}$ plus or minus the inerement $\Delta W_{i f}$. 
(a)

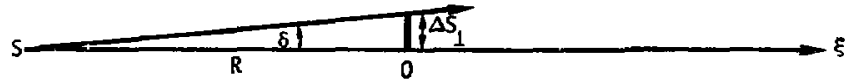

(b) $S \frac{W_{I}, v_{I} \longrightarrow}{\longrightarrow}-\xi$

(c)

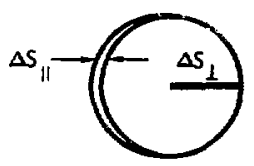

FIg. 3. Bearn divergence.

a) The spatial divergence due to ton optics. b) The focusing uncertainty in the longitudinal velocity direction. o) Disc formed at target site (from one source) due to both uncertainties.

$$
W_{\|}=W_{I} \pm \Delta W_{\|}=W_{I}\left(1+\frac{\Delta W_{\|}}{W_{I}}\right) .
$$

The veloctty then 1s approximately

$$
v_{\|}=v_{I} \sqrt{1+\frac{\Delta W_{U}}{W_{I}}}=v_{I}\left(I+\frac{\Delta W_{\|}}{2 W_{I}}\right)
$$


so that the longltudinal velocity uncertainty may be defined as

$$
\Delta v_{\| l} \triangleq v_{\| l}-v_{I}=\frac{v_{I} \Delta \omega_{\| l}}{2} \omega_{I} .
$$

A spatial uncertainty $\Delta S_{\|}$may now be defined in terms of the longitudinal velocity uncertainty $\Delta v_{l \mid}$ and tie characteristic flight time $t_{0} a s$

$$
\begin{aligned}
& \Delta S_{\|} \cong \Delta v_{\|} t_{0} \\
& \Delta S_{\|}=\frac{R \Delta W_{\|}}{2 W_{I}} .
\end{aligned}
$$

\section{FEASTBTLITY OF METEOD}

From the preceeding discussion, it is seen that the degree of density enhancement achievable will be deterwined by the uncertainties, In angle and in energy, assocfated with the nature of the neutral-beam sources, $i_{0} e .$, with the design and mechanical tolerances of the sources and with the initial thermal energy components of the accelerated ions. The dominant positional uncertainty is $\Delta S_{1}$. Thus, if the sources are located on a spherical surface of Iadius $R$, the heating energy will be concentrated witin a spherical shell region whose radius is of order $\Delta S_{\perp}$ and whose thickness is of order $\Delta S_{\|}$. Each elementary converged pulse bunch takes on the shape of a thin dfsc, as may be seen in Fig. 3(c).

An estlmate of the mean densi $y$ of the converged particles may now be made by recognizing that the bunching process has the effect of gathering up within a smaller spherical region of volume $4 / 3 \pi\left(\Delta S_{1}\right)^{3}$ an incoming pulse of particles that initially occupled a larger spherical volume. Thus, if the arerage surrent density of the neutral sources is $\mathrm{J}_{0}$, the mean density at converge-ıce may be estimated by dividing the total number of particles emitted Auring a pulse time, $t_{p}$, by the converged volume. 


$$
\bar{n}=\frac{\text { total particles emitted }}{\text { converged volume }} \approx \frac{4 \pi R^{2} J_{0} t_{p}}{4 / 3 \pi\left(\Delta s_{1}\right)^{3}} .
$$

For a 4:i enersy modulation, the pulse time is taken to be half the characterIstic flight tilne $t_{0}$. The mean de.rsity at convergence may then be rewritten as

$$
\bar{n} \approx \frac{3}{2 \sqrt{2}} J_{0}\left(\frac{M}{W_{I}}\right)^{1 / 2}\left(\frac{W_{I}}{\Delta \bar{H}_{\perp}}\right)^{3 / 2} .
$$

By taking current values for density and thermal energies from the existiag 50-A LBL source ${ }^{6}$ and assuming that similar values could be achieved in an energy-modulated spherical source array, the density of the converged particles may be calculated from the source parameters listed in Table 1.

Table 1. Source parameters asstered for system.

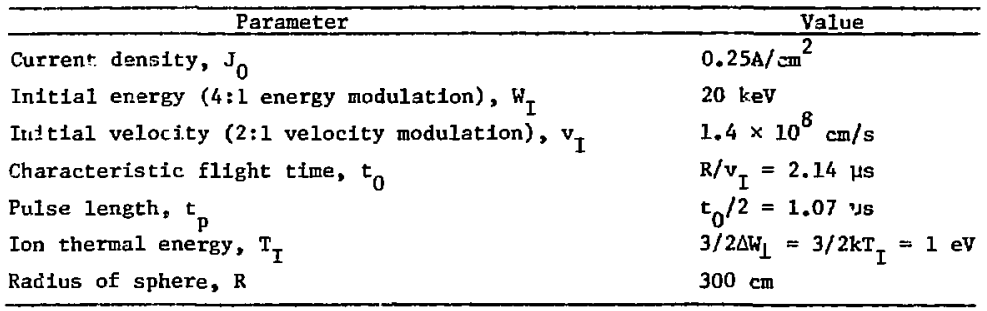

The feasibilicy of using this unique method of convergent neutral beams to create a high-density plasma at a target site may thereby be confirmed. For a deuterium beam (using Eq. 4), $\bar{n}=0.9 \times 10^{17} \mathrm{~cm}^{-3}$, a htgh density In the fusion context $(\beta=1$ at $30.0 \mathrm{~T})$. Furthermore, it should be possible to achleve substantially higher values by further source development. Owing to the short duration of the beam pulse, marked 1ncrease in both the emission current densf.ty and the beam eixergy may be possible. Under pulsed conditions, neutral-beam fluxes of several amperes equivalent per $\mathrm{cm}^{2}$ at beam energies of order $100 \mathrm{keV}$ seem attainable. ${ }^{9}$ Experiments with pulsed ion sources that suggest this possibility have been reported, Under these latter assumptions, the predicted densities are of order $10^{19} \mathrm{~cm}^{-3}$. 
In the next chapter, exact numerical solutions for this scheme will be dertved so that subsequent investigation and analysis can be made.

Additional pertinent characteristics and parameters of the proposed system may also be estimated from basic physical equations. Using the parameter values in Table 1, the streaming density at the source is

$$
\mathrm{n}_{0} \approx \frac{\mathrm{J}_{0}}{\mathrm{v}_{\mathrm{I}}}=10^{10} \text { neutrals } / \mathrm{cm}^{3} .
$$

A target site may be defined as a spherical region of radius $\Delta S_{1}$. Because of beam diverqence, all the energy of the neutral beams will be deposited within this spherical volume rather than on a point target. The mean volume of this region is

$$
\Delta V \approx 4 / 3 \pi \Delta s_{\perp}^{3} \approx 20 \mathrm{~cm}^{3} \text {. }
$$

The duration of this energy pulse $\Delta t$ may be simply estimated from the uncertainty parameter $\Delta S_{1}$.

$$
\Delta t=\frac{\Delta S_{1}}{v_{I}}=\frac{t_{0} \Delta S_{1}}{R}=t_{0} \sqrt{\frac{\Delta W_{\perp}}{W_{I}}} \approx 10 \mathrm{ns.}
$$

The power associated with the deposition of energy into this target sfte $1 \mathrm{~s}$

$$
P=\frac{\vec{n} W_{I}}{\Delta t}=\frac{10^{17} \cdot 20 \mathrm{keV}}{10 \mathrm{~ns}}=10^{10} \mathrm{~W} .
$$

\title{
Numerical Solution of Model
}

\author{
GENERAL APPROACH
}

To establish a formalism through which accurate predictions of the convergence-bunching processes can be made as they proceed, a computer code was written that predicts the evolution in time of a distribution finction 
$f(r, v, t)$. The initial source distribution function is taken to be a solution of the V1asov equation (derived in Appendix B) for a half-Maxwellian accelerated through the source electrode gtructure.

The code then follows the evolvement of the distribution function in time by using Liouville's theorem (Appendix C). It can tse the distribution function to get exact numerical solutions (within the uncertainties discussed In the previous chapter) for the convergence phenomena. In particular, the distribution function is necessary for calculating number densities, rate parameters, and tonization probabilities.

A major assumption made for this technique of using convergent neutral beams to create a transient, high-density plasma was that the beams are essentially noninceractive as they proceed toward the target site. The numerical solutions of the ionization probabilities for these beams show that this assumption of weak interaction is well verified.

Subsequent sections show the equations and calculations made. The general approach to the numertcal solution may be described as follows:

- Number density: The number density is found by numerical integration of the distribution function over approprlate velocity-space limits.

- Rate parameter: The rate parameters $\overline{\sigma v}_{\mathrm{NN}}$ and $\bar{\sigma}_{\mathrm{NI}}$ are calculated from a five-dimensional integration scheme over the velocity-space 11mits of the two reacting particles. The cross sections are tabulated in the code from curve fits to existing experimental data.

- Ionization probabllitles: These probabilities are calculated from the rate parameters through numerical integration up to a time of interest (corresponding to when the particles are said to be within the target site $\left(\rho \leq \Delta S_{\perp}\right)$.

DISTRIBUTION FUNCTION

The variation of the distribution during the acceleration process was derived as an exact solution to the Boltzman equation without collisions or forces. 
Figure B-1 shows the source distribution of the lons that result from acceler.tion through a potential $\mathrm{V}=\mathrm{Mv}_{0}^{2} / 2 \mathrm{e}$. After acceleration, the Ions are charge neutralized and emerge at the source with a directed velocity in the longltudinal direction much greater than the velocity components in the transverse directions. It is important to note that the source distribution that emerges is Gaussian in the velocity components perpendicular to the normal and approximately exponential in the longitudinal components. This characteristic of the distribution function, which is especially true for observation points greater than the uncertalnty $\Delta s_{\perp}$, will allow intuitive analytical approximations ${ }^{10}$ for the system of convergent neutral beams.

The distribution function at the source (Appendix B) is

$$
f_{s}(0)=\left\{\begin{array}{lc}
\frac{2 \alpha^{2}}{\pi} J_{0} \exp \left[-\alpha\left(v^{2}-v_{0}^{2}\right)\right] \text { for } v_{E}>v_{0} \\
0 \quad \text { otherwise. }
\end{array}\right.
$$

The neutrals will be emitted radially from the sources directed toward the center of the phere or cylinder. In terms of spherical coordinates, the radial componett of the velocity is $v \cos \theta$. The spherical coordinate system used in this analysis is defined as follows (see Fig. 1 ):

$R$ is the radius of the sphere, the distance from the source $S$ to the center

of the sphere 0 .

$r$ is the olistance from the source $S$ to an observation point $P$.

$\rho$ is the distance $F$ rom the center of the sphere 0 to an observation point $P$.

$\theta$ is the angle that the velocity vector makes with the normal.

$\eta$ is the spherical polar angle in velocity space.

$\xi$ Is the longitudinal coordinate (usual spherical coordinate $z$ ).

The modulating velocity will now be expressed in terms of an emission time, 1 ,e., the time when a particle would have to be emitted so that it could reach the observation point in a time $t$. The modulating velocity at the source had the dependence expressed in Eq. (1). The new dependence, defined in terms of the emission time $t_{e}=t-r / v$, is

$$
v_{0}(t-r / v)=\frac{v I}{1-\frac{(t-r / v)}{t_{0}}}=\frac{R}{t_{0}-t+\frac{r}{v}}
$$


The distribution function (using Liouville's theorum, Appendix c) translated In terms of these new coordinates has the fors

$$
f(r, v, t)= \begin{cases}\frac{M^{2} J_{0}}{2 \pi \Delta w_{1}^{2}} \exp -\left\{\frac{M}{2 \Delta W_{1}}\left[v^{2}-v_{0}^{2}(t-r / v)\right]\right\} & \text { for } v \cos \theta v_{0}(t-r / v) \\ 0 \text { otherw se. } & \text { and } 0 \leq(t-r / v) \leq t t_{p}\end{cases}
$$

Th1s expression is now the distribution function for a spherical geometry at the observation point $P$ and corresponds to Eq. (6) with

$$
\begin{aligned}
& t \longrightarrow t-r / v \\
& v_{\xi} \longrightarrow v \cos \theta .
\end{aligned}
$$

In doing the cylindrical problem, it wlll be show that it is possible to express these quantities in terms of varlables appropriate to a cylindrical coordinate system so that a simllar equation holds.

For an observation point far enough from the source, it is sufficiently accurate $t^{\prime}$ assume an effective uniform current density $J_{0}$ at the wa:l. The source is curned on for some pulse time $t_{p}$ and then curned off. Therefore, the particles are primarily located between some racil, $\rho_{\min }$ and $\rho_{\max }$, that correspond roughly to the postefons of thnse particles emitted at time $t=0$ at velocity $v_{I}$ and those emitted at time $t=t_{P}$ at velocity $v_{F}$.

For $\cos \theta=1, n=\pi$,

$$
\begin{array}{ll}
P_{\min }=R-v_{I} t & \left.v_{I}=v_{0}(t)=0\right) \\
P_{\max }=R-v_{F} t & \left.v_{F}=v_{0}(t)=t_{P}\right) .
\end{array}
$$

NURIER DENSITY

The number denalty for the ayetem of convergent neutral beams may be calculated by numertcally integrating over all velocity space; the density will simply be the first moment of the distribution function. 


$$
N(r, t)=\int_{a 11 \text { veloc.tty space }} \mathrm{f}(r, v, t) \mathrm{d}^{3} \mathrm{v}
$$

The integration schemes are given next along with an explanation of the numerical linits used for each integration.

\section{Spherical Geometry}

The velocity volume element in spherical coordinates (where azimuthal symmetry has been assumed) is $2 \pi v^{2}$ sinndndv. The expression to be integrated numerically is

$$
N(r, t)=2 \pi \int_{0}^{\pi} \sin \operatorname{dn} \int_{0}^{\infty} f(|v|, \eta, \rho, t) v^{2} d v
$$

The Interdependence of the vartables in real and velocity space (FIg. 1), can be derived from the law of cosines:

$$
\begin{aligned}
& \mu=\cos \eta=\left(\rho^{2}-\mathrm{R}^{2}+\mathrm{r}^{2}\right) /(2 \rho r), \\
& r=\rho \mu+\left[\mathrm{R}^{2}-\left(1-\mu^{2}\right) \rho^{2}\right]^{1 / 2}, \\
& \cos \theta=\left(\mathrm{R}^{2}+\mathrm{r}^{2}-\rho^{2}\right) /(2 \mathrm{r}) .
\end{aligned}
$$

Several thingó must be taken Into account when calculating the limits on speed $|v|$ and angle $\eta$ in velocity space. The primary motivation is to ascertain where most of the particles are in the distribution for any chosen position and time. Mathematical approximations based on the physics Involved w11l show the dependence of varlous parameters of the system. How these parameters vary in the distribution function will allow definition of approprlate limits of Integration.

From Eq. (8), note that the distribution function is zero inless

$$
\frac{r \cos \theta}{t}>v_{0}\left(t_{e}\right)
$$


There is a minimum velocity associated with the emission time $t_{e}=t-r / v$. The emission time is determined from what this time must be for particles to reach an observaition point in time $t$. If $\rho$ and $\mu$ are chosen, Eqs. (12) and (13) give $r$ and $\theta$.

If Eq. (14) Is satisfied,

$$
v_{\text {min }}=r / t
$$

Otherwise, the minimum velocity is calculated by solving Eq. (7) for $v=v_{\text {min }}$ " with the result

$$
v_{\text {min }}=\frac{R-r \cos \theta}{\left(t_{0}-t\right) \cos \theta}
$$

The maximum allowable velocity would be

$$
v_{\max }=r /\left(t-t_{p}\right)
$$

Particles wh greater velocities would have passed the observation point. These Ifmits are shown roughly in Fig. 4. In the actual numertcal integration over the veloclty variable $|v|, v_{\max } 1$ is determined by where the integrand drops off significantly so as to include only the pertinent area under the curve.

The integration over speed $|v|$ is

$$
F^{\prime}(\rho, n, t)=2 \pi \int_{v_{\text {min }}}^{v_{\max }} F(\rho, v, \eta, t) v^{2} d v
$$

The value of the Integrand may be defined as

$$
F(\rho, n, v, t)=f(\rho, v, n, t) v^{2}
$$

and evaluated at varlous points within the velocity limits. A trapezoidal numertcal integration for this integral is 


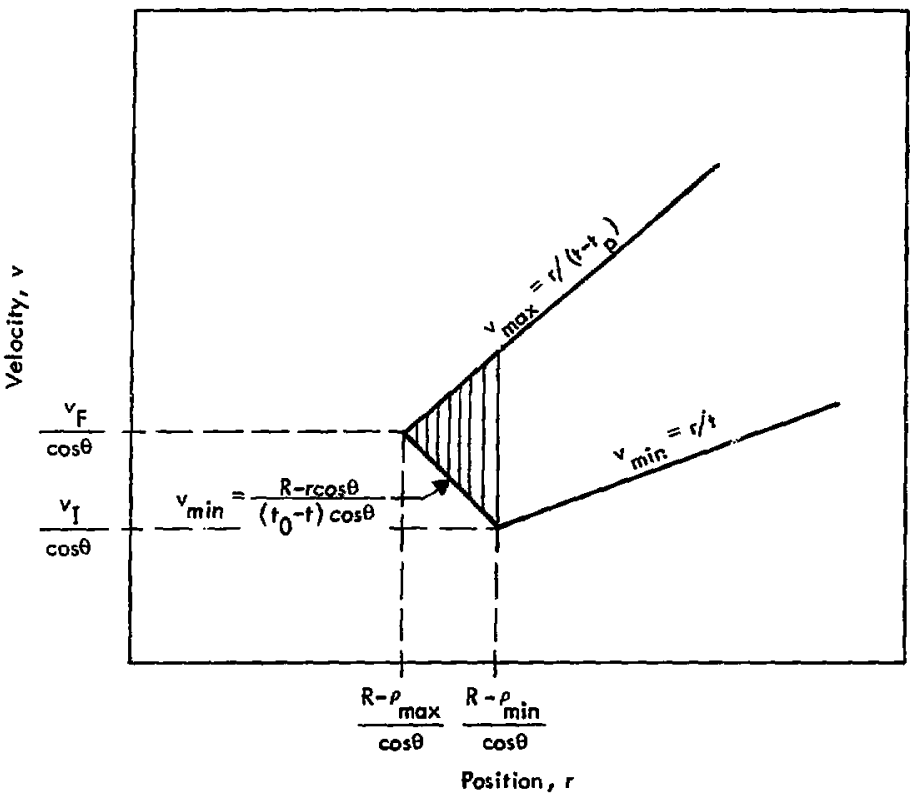
Fig. 4. The 11mits on velocity varlable $y$ for $t>t_{p}$, when $n \approx \pi$,
$\cos \theta=1, r=R-p$.

$F^{\prime}(p, n, t)=2 \pi d v\left[\sum_{N=1}^{N} F_{N}-\frac{F_{\max }}{2}-\frac{F_{N_{\max }}}{2}\right]$

where $N_{\max }$ is chosen to be a sufficlent numbar of integration steps (See Appendix E), and $F_{N}$ are values of the integrand for grid steps along $|v|$. A sample integration grid is shown schematically in Fig. 5. 
In calculating the 11dits on the polar veloctty angle in spherical coordinates, those regions that correspond to the physical location of tne particles as convergence is approached are considered. If appropriate approximations are made, ${ }^{10}$ analytical solutions may be found tor these regtons of interest. The approximate solutions and their dependence on the pertinent physical parameters of the system allow an accurate calculation of the 11mits needed for numerical integration.

An approximate form for the distribution function is given below:

$$
f=\left\{\begin{array}{l}
\frac{2 \alpha^{2} J_{0}}{\pi} \exp \left\{-\alpha\left[\frac{\mu^{2}\left(1-\mu^{2}\right) \rho^{4}}{\left(t_{0}-t\right)^{2} R^{2}}+\frac{2 \mu^{2} \rho^{2}\left(v-v_{\min }\right)}{\left(t_{0}-t\right) R}\right]\right] \\
\text { for } \geq \geq \rho_{\min } ; v_{\min } \leq v \leq v_{\max } ; \mu_{\min } \leq \mu \leq u_{\max } \\
0 \\
\text { otherwise. }
\end{array}\right.
$$

This approximation is readily integrated (See Appendix D) over the $|v|$ 1Imits $v_{\text {min }}$ and $v_{\max }$ with the result being

$F^{\prime}(\rho, \mu, t)=\frac{2 \alpha J_{0} R}{t_{0}-t} \exp \left\{-\alpha \frac{\left[\rho^{4} \mu^{2}\left(1-\mu^{2}\right)\right]}{\left(t_{0}-t\right)^{2} R^{2}}\right\}$ for $\mu_{\min } \leq \mu \leq \mu_{\max }$

$F^{\prime}(p, \mu, t) \approx 0$

otherwise.

Figure 6 shows how this function $F^{\prime}(\rho, \mu, t)$ varies with $\mu$, the cosine of the polar angle in spherfcal velocity space. At early times, the main part of the distribution is approximately a sharply decaytng exponential function of $\mu$. To calculate $\mu_{\max }$ here, the criterion is stipulated that the significant contribution to the integral is that portion where the integrand decreases by two orders of magniture. This truncation value is defined in terms of the parameters of the system:

$$
\mu_{L}=-\sqrt{0.5+\sqrt{0.25-I}} \text {, }
$$

where

$$
L \Delta \frac{4.614\left(t_{0}-t\right)^{2} R^{2}}{\alpha \rho^{4}}
$$




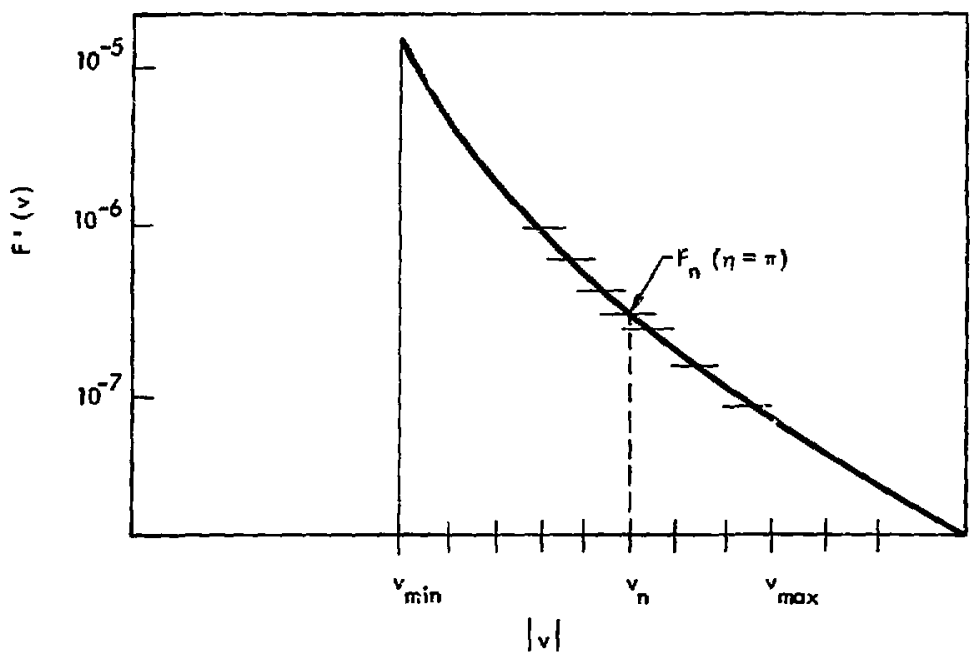

Ftg. 5. Sample Integration grid for numertcally calculating the integral over the veloctty varlable $v$, where $F^{\prime}(\rho, \eta, v, t)=K \exp \left[-\alpha\left(v^{2}-v_{0}^{2}\right)\right] v^{2}$.

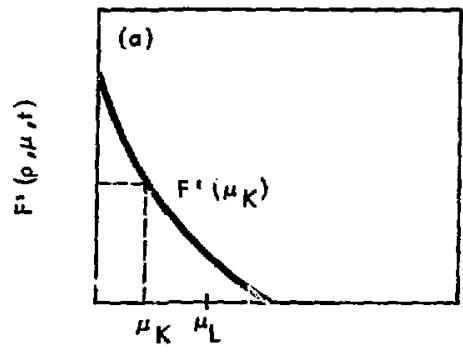

Cosine of velocity polar angle, $\mu$

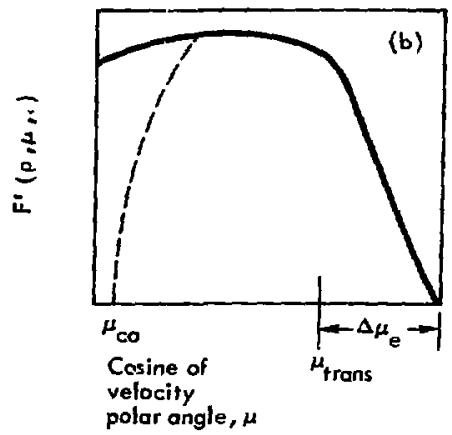

FIg, 6. Vartation of $F^{\prime}(p, \mu, t)$ with $\mu$.

a) Early time variation $\left(t \ll<-\rho \gg \Delta s_{1} ; \mu^{2}\left(1-\mu^{2}\right) \approx 2 \delta\right.$;

b) Vartation for times very close to convergence $\left(t+t_{0}, \Delta s_{\|} \ll \rho\right.$ $\left\langle\Delta S_{\ell}, F^{\prime} *\right.$ constant $=K \exp \left[-\alpha\left(v^{2}-v v_{0}^{2}\right)\right] v^{2}$. 
As time progresses, $1 . e .$, as the collection of particles proceeds towaxd convergence, this exponential broadens. When the particles get inside the convergence region, the distribution is mainly located between tro values of $\mu: \mu_{c o}$ and $\mu_{\text {trans, }}$ whjch are deterwined by the time $t$ and the position $p_{2}$ For these times near convergence, the distribution at any velocity $v$ is roughly constant between $\mu_{\text {co }}$ and $\mu_{\text {trans }}$. These cutoff points are determined by considering those angles at which all particles must have elther past or not yet arrived. Thus, $\mu_{c o}$ represents that value of $\mu=$ coen for which at sose chosen $p$ and $t$ all the particles emitted from the sources have gone by, $1 . e .$, when the velocity limits are equal. Equating Eqs. (16) and (17) and using Eqs. (12) and (13) to solve for this critical value of $p$ will give the following regult:

$$
\mu_{c 0}=\frac{\frac{-\rho_{\max }}{\rho}\left[1-\frac{\rho^{2}}{R \rho_{\max }}\right]}{\sqrt{1+\frac{\rho^{2}}{R^{2}}-\frac{2 \rho_{\max }}{R}}} \quad \text { for } \rho>\rho_{\max }
$$

and

$$
\mu_{c o}=-1 \quad \text { for } \rho<\rho_{\max }
$$

where $\mu_{\text {trans }}$ is a transitional point where the values of $F^{\prime}(\tau, \mu, t)$ start to drop off significantly. Here, the formula for $v_{\min }$ goes to $r / t$ instead of Eq. (16) and can be seen schematically in FIg. 6. The value of $\mu$ at this point is

$$
\mu_{\text {trans }}=\frac{\frac{-\rho_{\min }}{\rho}\left[1-\frac{\rho^{2}}{R \rho_{r},}\right]}{\sqrt{1+\frac{\rho^{2}}{R^{2}}-\frac{2 \rho_{m \text { mn }}}{R}}} \quad \text { for } \rho>\rho_{\text {min }} \text {, }
$$

and

$$
\mu_{\text {trans }}=-1 \quad \text { for } p<p_{\min }
$$


These two values, $\mu_{\text {co }}$ and $\mu_{\text {trans }}$, effectively limit the contribution to the distribution function. For purposes of accuracy, a small additional term $\Delta \mu_{\mathrm{e}}$ is also necessary to extend the integration domain beyond $\mu_{\text {trans }}$. The dependence of this term on the parameters of the system is

$$
\Delta \mu_{e}=\frac{4.614 R}{2 \alpha \rho v_{I}^{2}} .
$$

The 1imits for the spherical polar angle $(\mu=\cos \eta)$ in velocity space are listed beiow:

$$
\begin{aligned}
& \text { - For } L \leq 0.25 \\
& \text { and } \mu_{\text {trans }}+\Delta \mu_{e} \geq \mu_{L} \\
& \left\{\begin{array}{l}
\mu_{\min }=\mu_{\text {co }} \\
\mu_{\max }=\mu_{I}
\end{array}\right. \\
& \text { - For elther } \mathrm{L}>0.25 \\
& \text { or } \mathrm{I} \leq 0.25 \\
& \text { and } \mu_{\text {trans }}+\Delta \mu_{e}<\mu_{L} \\
& \left\{\begin{array}{l}
\mu_{\min }=\mu_{\text {co }} \\
\mu_{\max }=\mu_{\text {trans }}+\Delta \mu_{e}
\end{array}\right.
\end{aligned}
$$

Cnce the linits on 4 have been ascertalned, the integration itself is accomplished by using a 40-point Gaussian Integration scheme. The number density is then

$$
\begin{aligned}
N(\rho, t)=\int_{0}^{2 \pi} F^{\prime}(\rho, \eta, t) \operatorname{sinnan} & =\int_{-1}^{1} F^{\prime}(\rho, \mu, t) \\
& \approx \int_{\mu_{\min }}^{\mu_{\max }} F^{\prime}(\rho, \mu, t) \approx \sum_{k=1}^{N} a_{k^{\prime}} F^{\prime}\left(\mu_{k}\right) .
\end{aligned}
$$

The function $F^{\prime}$ is evaluated at the grid point $\mu_{k}$ and weighted by a factor $a_{k}$. The Gaussian integration constants are all scaled approprlately for the newly defined 11nits.

Approximate formulae for the number densities were also dertved 4 for regions of time pertinent to the system. By integrating Bq. (19) (assuming $n$ Is shaxply peaked about $\pi$ ), an approximate expression for the number density at early times is 


$$
N(\rho, t) \approx \frac{J_{0} R^{3}\left(t_{0}-t\right)}{\rho^{4}} \text { for } \rho_{\min }>\rho>\rho_{\max } .
$$

To further reinforce the validity of the approxirate and exact numerical calculations for early times, the system of convergent neutral beams was analyzed in macroscoplc terms. The resilts derived ${ }^{10}$ for the number densities at early times are independent of the distribution assumed for the statistical formulation in this report. This derivation is shown in Appendix $G$ for both the spherlcal and cylindrical geometrles. It was found that a whole famlly of acceptable solutions is possible.

At late times (times within the convergence region), the number densities vary as $1 / \rho$ between $\rho_{\max }$ and $\Delta S_{\perp}$. This may be seen by assuming that Eq. (19) is nearly constant between $\mu_{\text {trans }}$ and $\mu_{c o}$. The number density for this time region is

$$
N(\rho, t) \approx \frac{2 \alpha J_{0} R\left(\mu_{t \text { rans }}-\mu_{c o}\right)}{\left(t_{0}-t\right)} .
$$

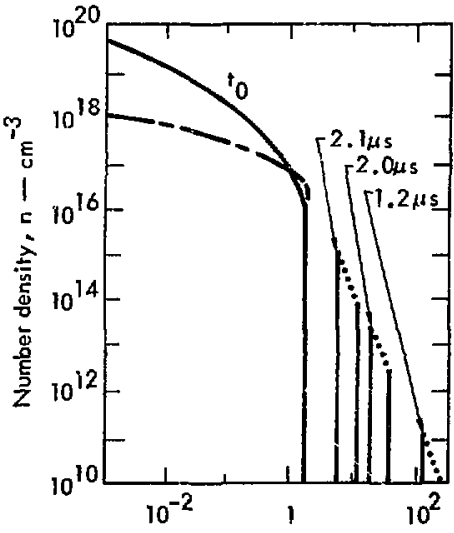

Distance from center, $r-\mathrm{cm}$

Fig, 7. Varlation of number density with distance from target site.

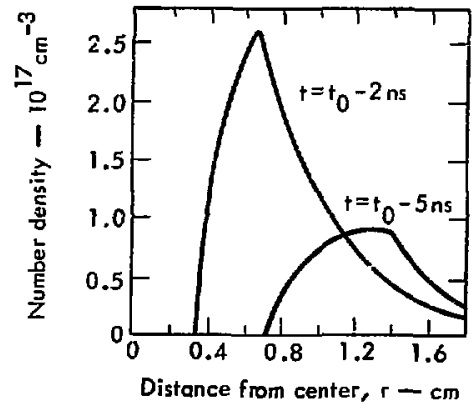

Fig, 8. Varlation of number density with distance from target site for last few nanoseconds. 
To 111 ustrate exact numerical result: obtained from the code. F1g. 7 shows on a log-log scale snapshots of the number density at successive time intervals during the convergence process. Figure 8 shows the last stages on an expanded linear time scale. The source parameters are the same as those given in Table 1.

In Fig. 7, a momentary sharp spike in density eppears to occur at the exact center. Actually, this spike is a result of the smaliness of the long1tudinal positional uncertalnty $\Delta \mathbf{S}_{\|}$of the target cloud. It would be expected that in an actual system, geometrical inaccuracies in the source system would substinfally weaken the spiking tendency. The dashed curve shown in Fig. 7 is a rough estimate of this effect for the case where the inaccuracies in source positioning at radius $R$ are about $\pm 0.5 \mathrm{~cm}$.

\section{CyIIndrical Geometry}

In doing the cylindrical problem, it was found that the parameters used In the spherlcal case could be expressed in terms of variables appropriate for a cylindrical coordinate system. A judicious redefinition of variables 10 allows the use of the same equations and coding for the distribution function as in the spherical case.

FIgure 9 shows a source position $S$ and an observation point $P$ defined In a typical cylindrical coordinate system. The source position is defined by $r, \theta$, and $z$. The projection of $r$ on the $\xi=0$ axis is defined es $s$. The velocity vector is defined by $v, \eta$ and $v_{z}$. Similarly, the projection of $v$ on the $\xi=0$ axis is defined as $v_{s}$. Ther by similar trangles, $r / s=v / v_{s}$, and $\mathbf{r} / \mathbf{z}=\mathbf{v} / \mathbf{v}_{\mathbf{z}}$

Given the coordinates of an observation point $\rho, n$, and $t$, the following relationships can be derived using these redefinitions:

- $\rho$ is now the distance along the $x$-axis from the center of the cylindrical coordinates system;

- I is the distance from the source to this observation point making an angle $\theta$ normal to the $\xi$ axis; and 


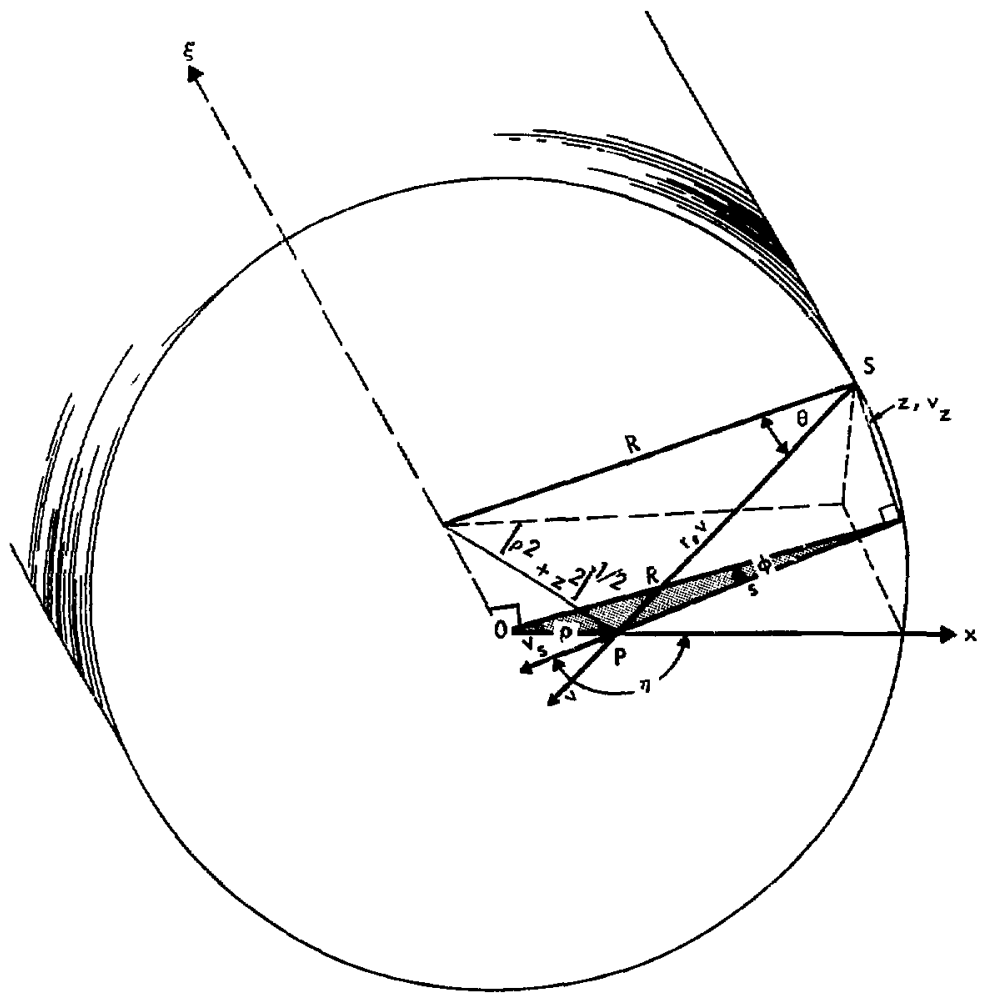

Fig. 9. Variables defined for a cylindrical cocrdinate system.

- $\eta$ is the angle $\rho$ makes with $s$ and is the cylindrical veloctty angle. Lookfing down the $\xi$-axis onto the $x y$-plane, the unknown variables can be solved In terms of the chosen parameters $\rho$ and $\eta$ using the law of cosines:

$$
\begin{aligned}
& \mathrm{R}^{2}=\rho^{2}+\mathrm{s}^{2}-2 \rho \mathrm{scog} \eta \\
& \rho^{2}=\mathrm{R}^{2}+\mathrm{s}^{2}-2 \mathrm{Rscos} \phi
\end{aligned}
$$




$$
\begin{aligned}
& \mu=\operatorname{cosn}=\frac{\rho^{2}+s^{2}-R^{2}}{2 \rho s} \\
& s=\rho \mu+\sqrt{R^{2}-\left(1-\mu^{2}\right) \rho^{2}}
\end{aligned}
$$

The code uses the same equations to calculate $s$ and $\phi$ as were us: for the coordinates in the spherical case ( $r$ and $\theta)$. From the right triangle made up of the vectors $I$ and $R$, a relationship for $\cos \theta$ in terms of $v_{s}$ is

$$
v \cos \theta=v_{s} \cos \phi
$$

The distribution function can now be defined for the cylindrical coordinate system. Liouville's theorem is again applicable for translating the definition of the distribution function to the observation point.

The velocity $l i m i t ~ v \cos \theta$ and the term $r / v$, which enters into the emission time $t-r / v$ in the spherical case, are now expressed in the cylindrical case in terms of the projections onto a plane normal to the axis of the cylinder.

$$
\begin{gathered}
r / v \longrightarrow s / v_{s} \\
v \cos \theta \longrightarrow v_{s} \cos \phi .
\end{gathered}
$$

The distribution function for the cylindrical geometry is

$$
f\left(p, v_{s}, n, v_{z}, t\right)= \begin{cases}\frac{2 \alpha^{2} J_{0}}{\pi} \exp \left[-\alpha\left(v_{s}^{2}+v_{z}^{2}-v_{0}^{2}\right)\right] \\ \text { for } v_{s} \cos \phi>v_{0}\left(t \cdots s / v_{s}\right) \\ 0 \quad \text { otherwise, }\end{cases}
$$

where the current density $J_{0}$ and the modulating velocity $v_{0}$ are evaluated at the cylindrical emission time, $t-s / v_{s}$. The subsequent integrations for the number denstty are done the same way as for the spherfcal case. 
Figure 10 shows the number density versus $\rho$ for different times and may be compared to a similat plot for spherical geometry (FIg. 7). Note that the densities are smaller than for the spherical case. The distributions look the same as they leave the source. In the sphertcal case, however, particles come in from both perpendicular directions, and the distribution gets broader in both perpendicular veloctly-space coordinates.

\section{IONIZATION PROBABILITIES}

In addition to calculating the number denstties anywhere Inside the contalnment sphere, other physical parameters of the system may also be calculated. Using the distribution functions and number densities already derived, the Ion densities, rate parameters, and lonization probabilities may be calculated for the converging beams. II

In calculating Ionlzation probabllitles, several assumpilons vere made:

- That neutral beams are nearly noninteractlve as they proceed toward the target site.

- That the form of the distribution function is not appreciably changed due to subsequent collisions when the beam is within the target site. This $1_{6}$ d reasonable assumption because the energy of the neutral atoms is great compared to the energy that they exchange durting collisions. The energy exchanged in such a reacclon is typically of the order of $10 \mathrm{ev}$, and the energy of the Incoming neutral is about $20 \mathrm{keV}$. It is therefore assumed that the Ion distribution function has the same form as the unperturbed neutral distribution function, but is reduced in magnitude by the radio of fon density to neutral density.

$$
\mathrm{f}_{1} \approx \frac{\mathrm{n}_{\mathrm{I}}}{\mathrm{n}_{0}} \mathrm{f}_{0} .
$$

Simflarly, the new neutral density will be reduced by the number of lons formed.

$$
f_{n} \approx \frac{\left(n_{0}-n_{I}\right) f_{0}}{n_{0}} .
$$


- That certaln terms in the rate equation could be neglected because their contribution is small. The equation used for the bulld up of fons 18

$$
\frac{\partial n_{I}}{\partial t}=n_{N}^{2} \overline{\sigma v_{N N}}+n_{N} n_{I} \overline{o v_{N I}} .
$$

The first term represents lonization by impact of two neutrais; and the second terw is due to the interaction between neutrals and ions.

The following sections wil show metheds and results achieved for the numertcal soluctons of the lonization probabilictes for these beans and wili verify the major assumption of noninteraction.

The general form for the rate parameter is a six-dinensional integral given by ${ }^{12,13}$

$$
\overline{\sigma v}=\frac{1}{n^{2}} \int d^{3} v \int d^{3} v^{\prime} \sigma\left(\left|v-v^{\prime}\right|\right)\left|v-v^{\prime}\right| f(v) f\left(v^{\prime}\right)
$$

The crosa sections, evaluated from a table of published experimental data ${ }^{14}$ are show In FIg. 11.

The probability that an atom wil be lonized in a time dt is

$$
\begin{aligned}
P(t) d t & =\left(n_{N} \overline{\sigma_{N N}}+n_{I} \overline{\sigma_{N I}}\right) d t \\
& =\left[\left(n_{0}-n_{I}\right) \overline{\sigma_{N N}}+n_{I} \overline{\sigma v_{N I}}\right] d t .
\end{aligned}
$$

If this equation is integrated over time, an lonization probability may be calculated.

$$
P_{\text {ion }}(t)=\int_{0}^{t} P\left(t^{\prime}\right) d t^{\prime}
$$




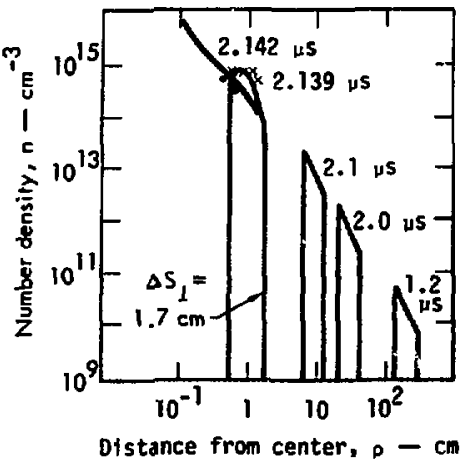

Fig. 10. Number density for a cylindrfcal coordinate systet as a function of distance from the center of the target site.

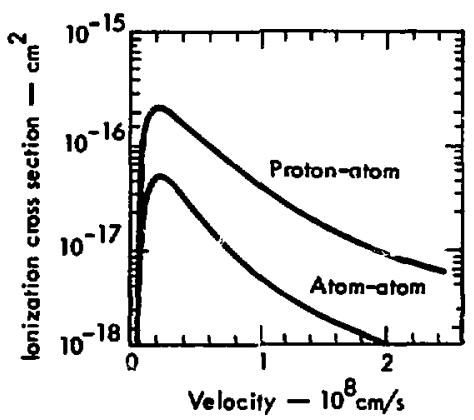

F18. 11. Aton-atom and proton-atom Ionization cross sections show as a function of the relattve velocity between the participating particles.

The number of lons formed at any time is given by

$$
n_{I}(t) \equiv n_{0}(t) P_{\text {Ion }}(t)
$$

This approximation wll remain true as long as the number of tons is much less than the number of neutrals and is [Eq. (27)] over-estimated by a fractional amount $n_{\mathrm{I}} / \mathrm{n}_{0}$ (see Appendix $\mathrm{F}$ ).

\section{Sphertcal Geometry}

Equation (31) shows the calculation necessary to find a solution for the rate parameter $\overline{\sigma v}$ for any process in a spherica.' configuration where azimuthal symatry exists. 


$$
\begin{aligned}
\left(\frac{}{\sigma v_{a b}}\right)_{s p h}=\frac{2 \pi}{n_{a^{n}} n} \int_{0}^{\infty} v^{2} d v & \int_{-1}^{-1} d \mu E_{a}(v, \mu) \int_{0}^{\infty} v^{\prime 2} d v^{\prime} \\
& \times \int_{-1}^{1} d \mu^{\prime} f_{b}\left(v^{\prime}, \mu^{\prime}\right) \int_{0}^{2 \pi} \sigma(u) u d \zeta .
\end{aligned}
$$

The usual angles of spherfcal ccordinate velocity space are contained in $\mu$ and $\zeta$, where

$$
\begin{aligned}
& \mu=\cos n^{\prime} \\
& \zeta=\phi-\phi^{\prime} .
\end{aligned}
$$

This reduces a six-dimenstonal integration In veloclty spase to a fivedimenstanal one. The form of ine relative velocity in these coordinates is

$$
u=\left[v^{2}+v^{\prime 2}-2 v v^{\prime}\left(\mu \mu^{\prime}+\sqrt{1-\mu^{2}} \sqrt{1-\mu^{\prime 2}} \cos \zeta\right)\right]^{1 / 2}
$$

Figure 12 shows a plot of the rate parameters versus time for a spherical geometry where $\rho$ was chosen to be near the front edge of the neutral-beam pulse. This $\rho$ corresponds to a position of the neutral particles at a particular time assuming they left the source first with the slowest velocicy.

Figure 13 shows the Integration of the rate parameter to get the Ionization probability $[E q,(29)]$. As convergence is approached, the fonfzation probability increases rapidly. This increase is due in part to the Increase In the relative velocities of the particles as they approach convergence (which leads to an increase in the cross section untll it levels off). There is also a rapid increase in the number denst ty as convergence is approached. For a point in the distrioution near the front of the pulse, $10 \mathrm{~ns}$ before convergence, only $3 \%$ Ionization occurs. 
Figures 14 and 15 show the rate parameter and fonization probabilities for a position corresponding to the back of the neutral-beam pulse. For this point in the distribution, as the pulse collapses toward the target, the probabilities become larger because the velocfties are larger ( $80 \mathrm{keV}$ instead of $20 \mathrm{keV}$ in the test case). Very close to convergence, the probability approaches one; however, the formulas that were use (Appendix F) overestimate this value.

\section{Cylindrical Geometry}

The rate parameter and relative velocity for a cylindrical geometry are

$$
\begin{aligned}
n_{a b}{ }_{b} \overline{\sigma v} & =\int_{a b}^{\infty} v_{s} d v \int_{s}^{2 \pi} d n \int_{0}^{\infty} v_{s}^{\prime} d v_{s}^{\prime} \int_{0}^{2 \pi} d n^{\prime} \int_{-\infty}^{\infty} d v_{z} \\
& \times \int_{-\infty}^{\infty} d v_{z}^{\prime} f_{a}\left(v_{s}, \pi, v_{z}\right) f_{b}\left(v_{s}^{\prime}, n^{\prime}, v_{z}^{\prime}\right) \sigma_{a b}(u) u, \\
u & =\left[v_{s}^{2}+v_{s}^{\prime 2}-2 v_{s}^{v_{s}^{\prime}}\left(\cos n \cos n^{\prime}+\sin \sin n^{\prime}\right)+\left(v_{z}-v_{z}^{\prime}\right)\right]^{2 / 2}
\end{aligned}
$$

The rate equation is again a six-dimensional integral. The velocity-volume coordinates considered now have a $v_{2}$ component. A judiclous transformation of varlables and redefinitions ${ }^{10}$ again reduces the integrations required to five by defining new vartables as the sum and difference of the directed velocity component.

$$
\begin{aligned}
& w=\frac{1}{\sqrt{2}}\left(v_{z}+v_{2}^{\prime}\right) \\
& z=\frac{I}{\sqrt{2}}\left(v_{z}-v_{z}^{\prime}\right)
\end{aligned}
$$

The product of the distribution functions $f_{a b}$ is then proportional to $\exp \left[-\alpha\left(w^{2}+z^{2}\right)\right]$. 


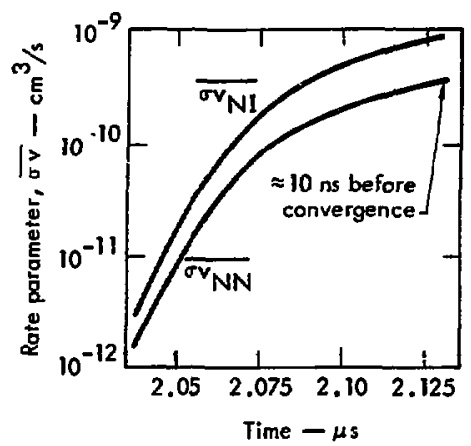

Fig. 12. Rate parameter versus time for a spherical geometry $\left(\rho=1.05 p_{\min }\right)$ near the front of the beam pulse.

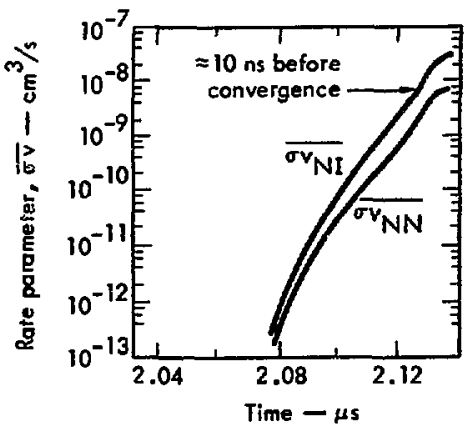

F1g. 14. Rate parameter versus time for a spherfcal geometry $\left(\rho=0.975 p_{\text {max }}\right)$ near the back of the beam pulse.

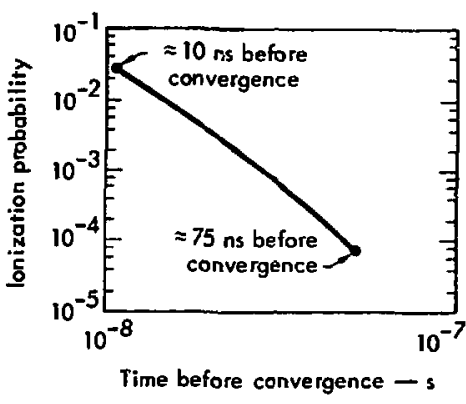

F18. 13. Ionfzation probabllity versus time for a sphertcal geometry $\left(\rho-1.05 \rho_{\text {min }}\right)$ near the front of the (beam) pulse.

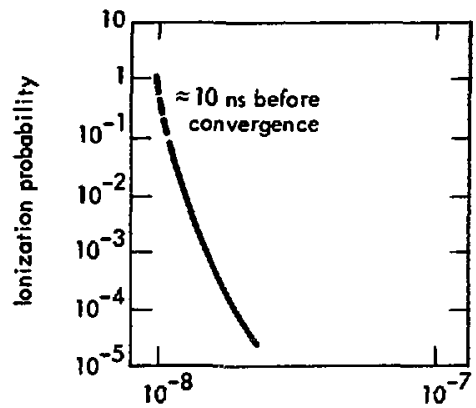

Time before convergence - s

FIg. 15. Ionization piobability versus time for a spherical geometry $\left(\rho=0.975 \rho_{\text {max }}\right)$ near the back of the beam pulse. 
Since $w$ doesn't enter Into the relative velocity $u$ and is only contained in the distribution function, the exponential portion $e^{-\alpha w 2}$ can be taken out and integrated separately, giving the factor $(\alpha / \pi)^{1 / 2}$. The rate parameter in terms of these variables is

$$
\begin{aligned}
& \pi_{a b b} \overline{\sigma v_{a b}}=\sqrt{\frac{\alpha}{\pi}} \int_{0}^{\infty} v_{s} d v_{s} \int_{0}^{2 \pi} d \eta f_{a}^{\prime \prime}\left(v_{s}, n\right) \int_{0}^{\infty} v_{s}^{\prime} d v_{s}^{\prime} \\
& \times \int_{0}^{2 \pi} d n^{\prime} f_{b}^{\prime \prime}\left(v^{\prime}, n^{\prime}\right) \cdot 2 \int_{0}^{\infty} d z e^{-\alpha z^{2}} \sigma(u) u
\end{aligned}
$$

The formalism of the code for the case of a cylindrical geowetry is the same with the exception of a slightly different $u$ and a different weight function in the integrand. The volume element now contalns $v_{s} d v_{s}$ instead of $2 \pi s i n \eta$.

Figure 16 showg the rate parameter $\overline{\sigma v}$ versus time for cylindrical coordinates. The same values of $p$ as in the spherical case were chosen for comparison.

Figure 17 shows the lonization probabilities for a $p$ near the front of the neutral-beam pulse. At 10 ns before convergence, the probability $180.02 \%$. This probabilfty is smaller than for the spherical case. The distribution looks the same as it leaves the source, but the lonization rate is less in the cyliudrical case. In the spherical case, particles cotre in from both perpendicular directions, and the distribution gets broader in both perpendicular velocity-space cnordinates. The density is less in the cylindrical case due to this loss of one dimension.

Figures 18 and 19 show the same information for a point in the distribution toward the back of the beam. The same statements hold as for the spherical case. The particles emitced later have a higher velocity, so the degree of loniaation $10 \mathrm{~ns}$ before convergence $(0,2 \overline{7})$ is increased.

\section{CONPUTER COUE}

The code is writgen for use on the CDC 7600 computer and is written in the LRLFRAN version of the FORTRAN IV language. 


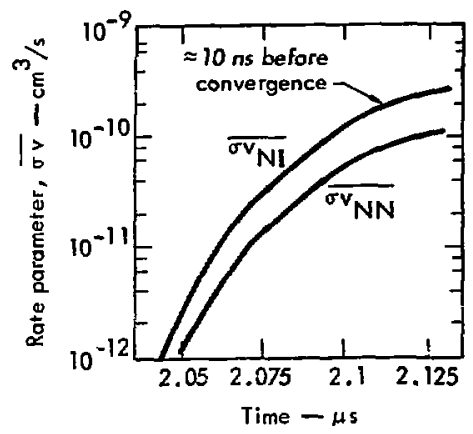

Fig. 16. Rate parameter versus time for a cylindrical geometry $\left(p=1.05 p_{m 1 n}\right)$ for the front of the beam pulse.

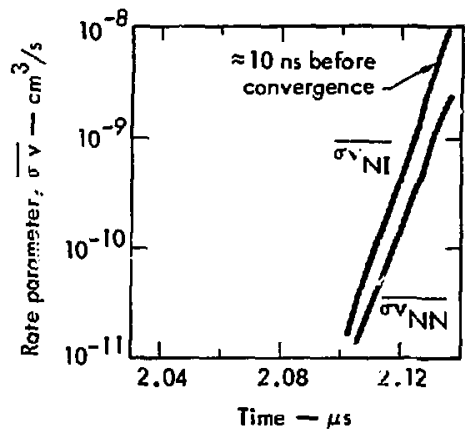

FIg. 18. Rate parameter versus time for a cylindrical geometry $\left(\rho=0.975 \rho_{\text {max }}\right.$ ) at the back of the beam pulse.

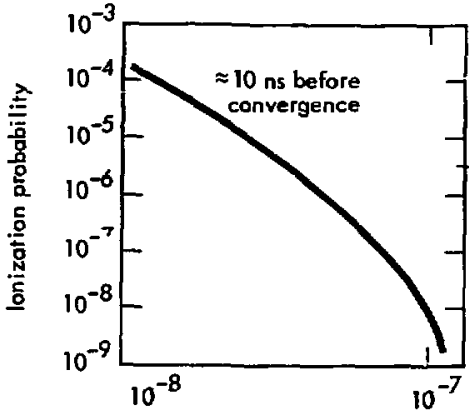

Time before convergence - 5

F1g. 17. Ionization probability versus time for a cylindrical geonetry $\left(\rho=1.05 \rho_{\text {nin }}\right)$ at the front of the beam pulse.

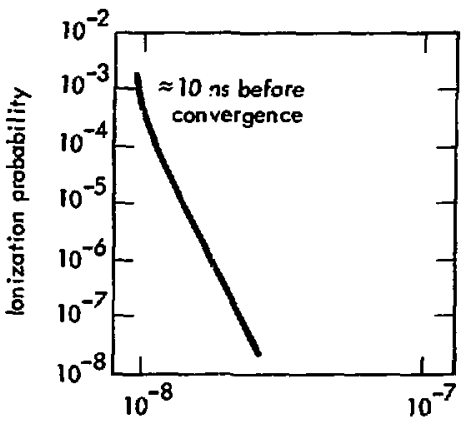

Time before convergence -5

Fig. 19. Ionization probability versus rimr for a cylindrical geometry $\left(p=0.975 \rho_{\max }\right)$ at the back of the beam pulse. 
The code occuples approxtmately $(40000)_{a}$ core locations. The code runs fully in core, but could be easily modified to run using dise storage shotid subsequent versions require $1 \mathrm{t}$.

Running time on the $\operatorname{CDC} 7600$ for a sample problem was 3 minutes. The timing depends strongly on the number of steps taken in each of the six nestyd integration loops for the numerical calculation of Eq. (29). In the flos chart of the code, Fig. 20, the optimum number of integration steps is carked for each lcop. By ralsing the number of steps until no differences appeared In the answer, it was possible to guarantee that sufficient integration steps were used for each integral. However, a more accurate analytical method was derivej $^{10}$ using the error cerm for trapezoldal integration (see Appendix $E$ ).

\section{Discussion of Plasma Creation}

Thus far In this report, no predictions have bcell made absut the subsequent history of the newly created plasma. It is hoped that the euerzy w11 be deposited at the target site before scattering and that ionization effects could appreciably influence the final convergence process. However, probiems may arise from electron heating, the creation of self-electromagnetic fields, and the onset of instabilities. These possibilities wll now be briefly discussed.

Calculations of the ionization probabilities ignored various additional mecitunisms such as electron heating that could become important as the ion and electron densities increase. Newly created ions have nearly all the energy of the parent neutral; the electron has little energy $(\approx 10 \mathrm{eV})$. How ever, once ionfzation has occurred, the energy gained by the electron between collisions can produce additional ionizations via a cascading effect. 15

Analysis of the subsequent history of the newly created electrons is done by examining the electron transport equations. These equat tons are derived by Braginskii by taking the moments of the Boltzman equation ${ }^{16}$ and incorporating the conservation (mass, momentum, energy) laws.

The electron transport equations delineating the important mechanisms pertinent to this problem 8 are listed below. 
For a chosen $p$ (i.e., $p=p_{\min }$ ), calculate appropriate integration limits for each time; calculate relative velacity, rate parameters, ionization probabilities, ion and neutral densities by sequential numerical integrations.

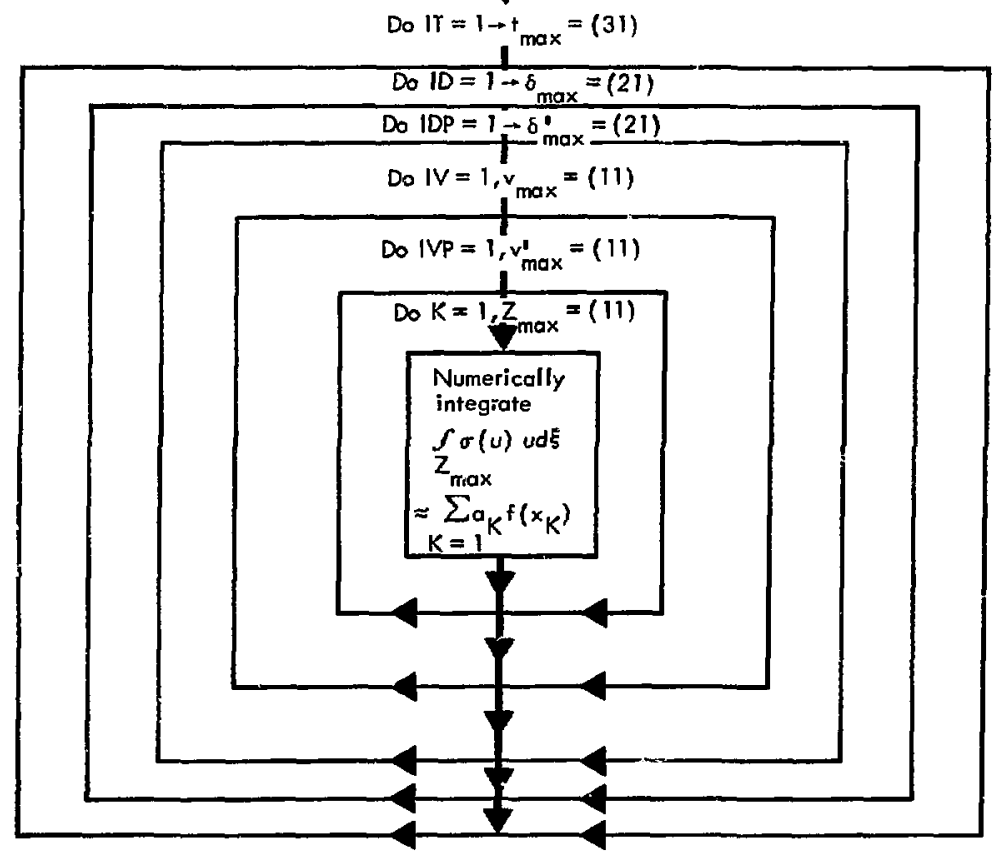

F1g. 20. Flowchart of code to calculate various parameters of convergent neutral beans.

$P(t)=\int d t \int d \delta \int d \delta^{\prime} \int f v^{2} d v \int f^{\prime} v^{\prime 2} d v^{\prime} \int \sigma(u) u d \xi$, where u is a furetion of $t, v_{,} v^{\prime}, \delta, \delta^{\prime}$. 
Mass transport:

$$
\frac{\partial n_{E}}{\partial t}+\operatorname{div}\left(n_{E} v_{E}\right)=S_{E}
$$

Momentum transport:

$$
m_{E} n_{E} \frac{d v_{E}}{d t}=-\frac{\partial P_{E}}{\partial x}+e n_{E} E+R,
$$

Electron energy transport:

$$
\frac{3}{2} n_{E} \frac{d T_{E}}{d t}+P_{E} \operatorname{div} v_{E}=Q_{E}-\frac{3}{2}{ }_{i} S_{E} \text {. }
$$

The variables in Eqs. (36) through (38) are: 16

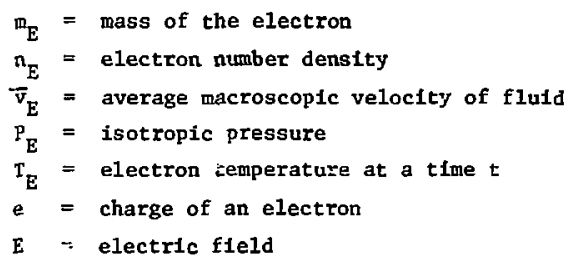

The electron energy transport equation neglects terms representing antsotropic pressures, radiation, thermal conductton, and the effect of a magnetic field. If $\lambda_{\text {Debye }}<\rho$, as in this case, the electrostatic and pressure gradient forces on the electrons must be closely in balance. The electrons, being ilght and moblle, would be accelerated to high energles very quickly if there were a net force on them. If the electrons left a region, a large Ion charge would remain, and the plasma tendency toward charge neutralfty would be violated. The slight separation of charge gives rise to an electric fyeld that binds the electron cloud about the fons so that the electrons are constrained to wove with them. 17

The source term $S_{E}$ zepresents the fategral over velocity space of the Inelastic collision term in the Boltzman equation.

$$
s_{E}=\sum_{\substack{\text { all } \\ \text { species }}} c_{\alpha \beta} d v \text {, }
$$


where $C_{\alpha \beta}$ describes any possible interaction process between species $\alpha$ and species $\beta$.

In a partially ionized plasma, the three constituents (electrons, fons, and neutrals) can produce subsequent lonizations via the following processes:

$$
\begin{aligned}
& n+\mathrm{n} \longrightarrow 2 \mathrm{H}+2 \mathrm{e}, \\
& \mathrm{n}+\mathrm{e}-\mathrm{H}+2 \mathrm{e}, \\
& \mathrm{n}+\mathrm{H}-\longrightarrow \mathrm{H}+\mathrm{e}
\end{aligned}
$$

Therefore,

$$
s_{E}=n_{N}^{2} \overline{\sigma v}_{N N}+n_{N} n_{I} \overline{\sigma v}_{N I}+n_{N} n_{E} \overline{\sigma v}_{N E}
$$

Vartous other possible mechanisms are neglected because the rate parameter $\overline{\sigma v}$ is small in the energy range considered here.

The quantity $R$ represents the mean change in the momentum of the electrons due to colltsions with all other particles.

$$
R=\sum_{\substack{\text { all } \\ \text { spec1es }}} \mathrm{mv}^{\prime} \mathrm{c}_{\alpha \mathrm{B}} \mathrm{dv} \text {. }
$$

The rate of electron heat generation $Q_{E}$, resulting from collisions between the constituent particles represents the following processes:

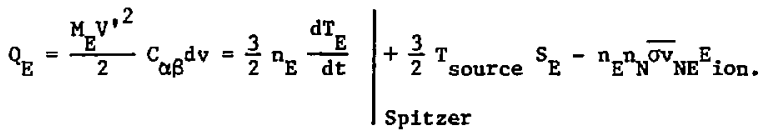

The first term on the right-hand side is the Spitzer heating term ${ }^{18}$ due to int ractions between the ions and the electrons. The second is the effect on the temperature rate due to electrons being born at a low energy ( $T_{\text {source }}$ is assumed to be $06.8 \mathrm{eV}$ ). The last term is a cooling effect on the electron temperature rate since the electrons lose energy when they cause an ionization. The energy required to form an $10 \mathrm{p}$ pair, $E_{10 n}$, is $40 \mathrm{eV}$, and includeg various processes (suct, as excitation) for possible interactions between neutrals and electrons. 
The electron pressure $P_{E}$ was considered isotropic since the electrons thermalize (to a Maxweilian distribution) quickly and set equal to $n_{E} k T_{E}$. The expression used for the heating of electrons due to the compression term $\left(P_{E}\right.$ div $\left.\bar{v}_{E}\right)$ in the spherical geometry is

$$
P_{E} \operatorname{div} \bar{v}_{E}=n_{E} k T E\left[\frac{1}{\rho} \frac{\partial}{\partial \rho}\left(\rho^{2} \bar{v}_{E}\right)\right] \text {. }
$$

Using the approxination for early times or

$$
\begin{aligned}
& {\overline{v_{E}}}_{E}-\frac{\varphi}{\varepsilon}, \\
& \frac{2 P_{E} d i v \bar{v}_{E}}{3 n_{E}}=-\frac{2}{\varepsilon} T_{E}
\end{aligned}
$$

Eq. (38) then becomes,

$$
\frac{d T E}{d t}=\frac{2 Q_{E}}{3 \pi_{E}}-\frac{T_{E} S_{E}}{n_{E}}+\frac{2 T_{E}}{\varepsilon},
$$

or

$$
\frac{d T_{E}}{d t}=\frac{d T_{E}}{d t_{S p i t z e r}}+\frac{T_{\text {source }} S_{E}}{n_{E}}-\frac{2}{3} n_{N} \overline{\sigma v}_{N E} E_{\text {ion }}-\frac{T_{E} S_{E}}{n_{E}}+\frac{2 T_{E}}{E} .
$$

An equilibrium temperature for which the electron temperature rate was zero was calculated from Eq. (41) and was assumed to provide an upper bound for any position and time. The rate parameter $\overline{\sigma v}_{N E}$ was calculated for this temperature, and the ionizations due to lons was compared to the Ionizations due to electrons. Within the constrafints of the assumptions used (Including a perhaps overly conservative estimate of $\sigma \mathrm{v}_{\mathrm{NE}}$ ), some pertinent parameters of the system of convergent neutral beams were made for interesting times and positions. These are enumerated in Table 2. Note that even where the rate parameter $\overline{\sigma v}_{\mathrm{NE}}$ is greater than $\overline{\sigma v}_{\mathrm{NI}}$, the ionization rate due to neutralneutral interactions is always higher than the electron (or fon) rates, at least up to those times defined to be within the target site. It is therefore hoped that the degree of Ionization due to electrons would remain small up to that point $\Delta S_{1}$ where the convergence process has been essentially completed. There would not be enough time left for any subsequent mechanism to appreciably alter either the final density or the convergence of the beam. 
Table 2. System parameters as a function of time and position.

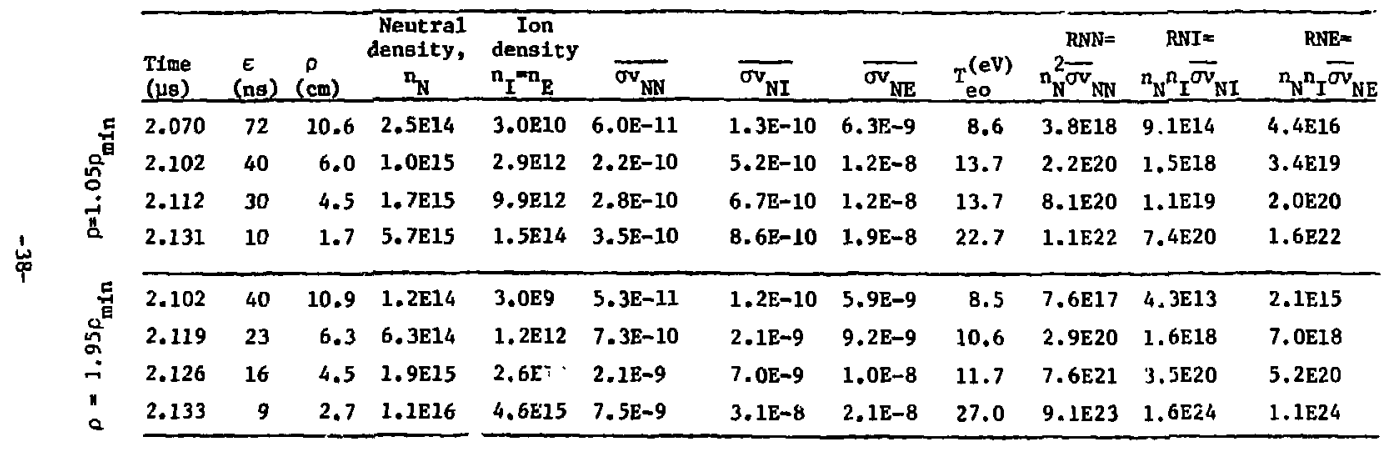


Another consequence that may possibly occur within the target site due to the creation of a plasma is the growth of Instabilities. These would affect the Ionization rates. The Buneman tworstream and Weibel Instabilities ${ }^{8}$ seem to have growth vates greater than the lonization rate. The creation of self flelds could give rise to a plethora of plasma Instabilities. Accurate calculations of these growth rates could be done in the program using the distribution function. Since the distribution function becones more isotropic as :Ime goes on, it is hoped that this would help to damp out the Instabilities and that the energy of the neutral-beam pulse would be deposited at the target site unimpeded.

Addftionally, subsequent analysis $n$ : the newly created plasma "ould examine the effects of external magnetic fields. These would help to confine the plasma as the neutral cloud turns into plasma. The B 1ines would be frozen in as the nonconductor became a conductor, thareby counteracting the plasma pressure attempting to push the charged particles to the wall.

\section{Summary}

On the basis of the analysis and calculations made in this proposal and in view of advances in high-current, pulsed neutral-beam technology, it is belleved that the convergence-bunching technique could have important application In fusion power research.

Some possibilities for using the high power denstties and high Instantaneous momentum fluxes assoclated with the converging neutral beams, could have important applicaticns in fussion power research. For example:

- High-beta or reversed-field states in intense magnetic fields might be created on a nanosecond time scale. Some such states appear to be intrinsically Inaccessible via cunventional methods where plasmabuildup times are long compared to particle losk times.

- In high-beta regions, transients may be stabilized againgt the onset of Instabilities or a potcntial well could be created so that electrons are trapped in the well as the fon density increases.

- In a cylindrical geometry, a momentum-stabilizing pinch could Inhibit the kink instability.

- In tokamaks, It may be possible to overcome the linitations of ohmic heating and heat a plasma to Ignition. 
- For inertial confinement, the method might be used as an alcernative to laser, electron, and Ion beam schemes. Radially convergent neutral beams in efther spherfeal or cylindrical geometry would be used to compress a deuterium pellet.

other possibilfties for utilizing the high peak power densities and high Instantaneous momentum fluxes that would be associated with such converging beams wil no doube be proposed as this technique is brough to practical realtzation. 


\section{Acknowledgments}

The assistance provided by tha following people was Invaluable:

Richard F. Post for the initial conception of this proposal and for continuous consultation and encouragement.

Kenneth Marx for his continual guldance and patience and formulation of the equations and approximations.

Charles Hartman and Dan Shumaker for Insight, advice, and significant moral support. 


\title{
Appendix A. Characteristics of Neutral Beams
}

\author{
SPACE CHARGE
}

One of the fundamental processes which limtt the collimation of ton beams is space charge. The law for apace-charge-limited current between parallel places implies that for a given voltage the current flowing from a source to as accelerating electrode w111 not increase beyond a limiting current density. This forms a fundamental 11mitation on the ton current atcainable from an ion source. The space-charge-limited current between two para1lel plates is proportional to

$$
\mathrm{J} \propto \frac{\mathrm{v}^{3 / 2}}{z^{2}}
$$

where $V$ is the potential difference, and $z$ is the distance between the two plates. The Ion flow between two parallel electrodes is shown in Fig. A-1. From proportionality, it can be seen that the attainment of a large ion current, as desired by fusion resea:ch, requires n small electrode spacing. This consideration and the desire to obtain a low-divergence Ion beam motivated the use of a large plasma surface with multiaperture or gridded electrodes.

\section{PLASMA CHARACTERISTICS}

The plasma density at the extraction surface must be uniford ispace and time over the period of interest and must be free of oscillations. From the space-charge Eq. (Al), the current density depends on the spailng between the electrodes. A spacing that is too small is Impractical to obtain at high energles; therefore, the Ion density must be reduced by allowing the plasma to expand to a larger volume. Many systematic studies have been undertaken wth the object of controlling plasma diffusion to imprcte the optical qualities of the extracted fon beam. 
The shape and uniformity of the plasma menicus is important in the net focusing of the beam. A slightly concave meniscus of homogeneous plasma with untform curvature has achleved ton beams in which the tivergence is comensurate with the temperature of the plasma $\left(\Delta \theta=T_{1}\right)$.

\section{ELECTRODE SHAPE}

To exti 3ct and accelerate beams of maximum intensity and minimum divergence, electrodes having good ton optics must be used. Focusing is accomplished by having lens action balanced against space-charge forces to achleve parallel flow from an inwardly focused beam.

Figure A-2 shows models for the behavior of shaped and unshaped extraction systems. 19 Note that the shaped electrode minimizes the divergence of the beam.

\section{NEITTRALIZATION OF ION BEAM}

Space-charge neutralization may be actalned by adding electrons across the entire cross section of the beam. The effictency of the process is energy dependent; the efficlency in neutralizing charge particles decreases as thelr energy increases, Accel-decel electrode systems are utilized to extract these beams and simuitaneously to act as an electrostatic trap for electrons. Calculations, done by computer, can predirt optimum size and spacing of these apertures. 


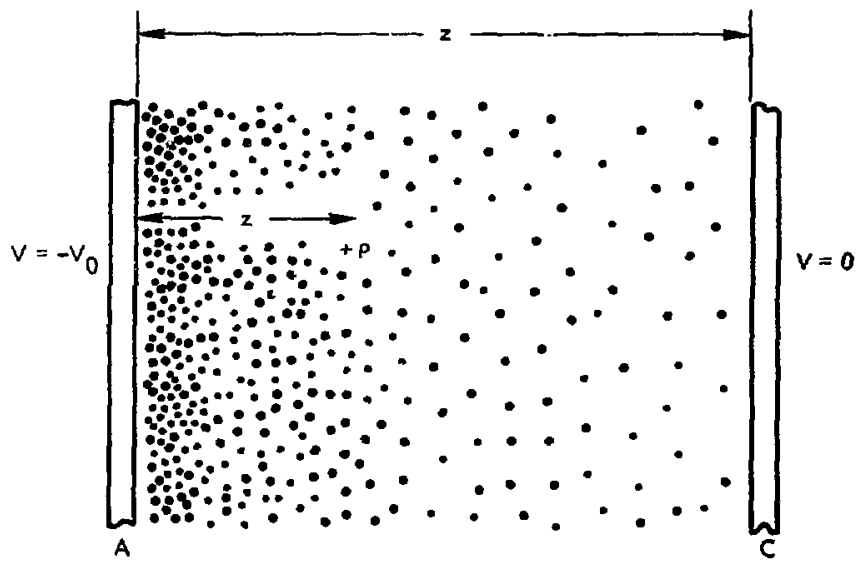

F1g. Al. Space charge between two parallel electrodes.

(a)

(b)
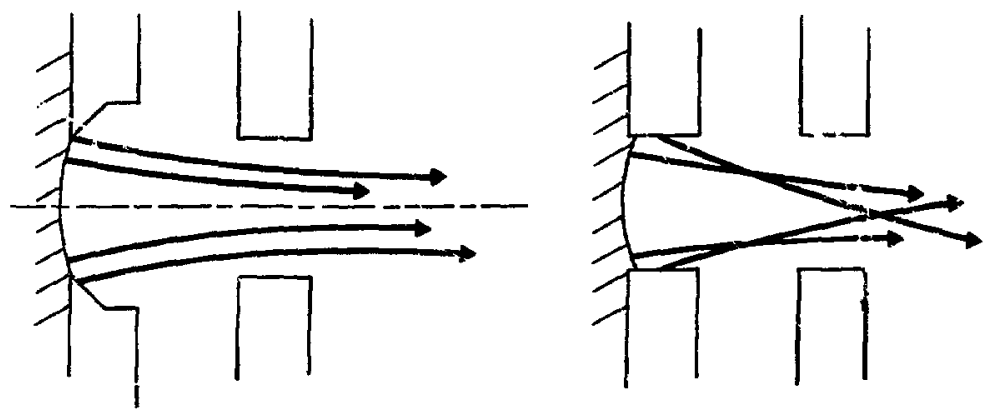

F18. A2. Models for behavior of (a) shaped and (b) unshaped extraction systems. 


\section{Appendix B. Solution to Vlasov Equation Within an Ion Source}

The variation of the distribution function in phase space as it travels through the fon electrode structure is given by the Boltzman equacion (or Vlasov equation for no collistons).

The steady-state, one-dimensional Vlasov equation for this problem is

$$
\mathbf{v}_{\xi} \frac{\partial \mathbf{f}}{\partial \xi}+\frac{\mathrm{eE}_{\xi}}{\mathrm{M}} \frac{\partial \mathrm{f}_{\xi}}{\partial \mathbf{v}_{\xi}}=0
$$

The assumption is made that the electric field $E(\xi, t)$ is a slowly varying (nearly constant) quanticy. An thitial distribution is hypothesized at the entrince of vise acceleration region to be a half-Maxwellian. This assumes that there are no backwards-emerging lons from the plasma source. If the distribution function can be writcen In terms of the constants of motion, the distribution function after acceleration (the solution to the vlasov equation) is defined.

This will now be shown to be true for this problem, where the constant of motion is $v_{x}^{2}, v_{y}^{2}$, and

$v_{\xi}^{2}=\frac{2 e}{M} E\left(\xi+\xi_{a}\right)\left[v_{x}^{2}, v_{y}^{2}, v_{\xi}^{2}-\frac{2 e E}{M \alpha}\left(\xi+\xi_{a}\right)\right]=$ const.

Therefore,

$$
\begin{aligned}
f & =A \exp \left(-\alpha\left[v_{x}^{2}+v_{y}^{2}+v_{\xi}^{2}-\frac{2 e E}{H a}\left(\xi+\xi_{a}\right)\right]\right) \\
& =A \exp \left[-\alpha\left(v^{2}-v_{0}^{2}\right)\right] .
\end{aligned}
$$

The lons emerge diter acceleration with an additional energy component in the longitudal direction $(\xi)$ equal to $2 \mathrm{eV} / \mathrm{M}$. Then upon emergence, the infeial variables can be rewritten as

$$
\begin{aligned}
& v_{x}^{v^{2}} \longrightarrow v_{x}^{2} \\
& v_{y}^{\prime 2} \longrightarrow v_{y}^{2} \\
& v_{\xi}^{\prime 2} \longrightarrow v_{\xi}^{2}-2 e v / M=v_{\xi}^{2}-\frac{2 e E}{M} \xi .
\end{aligned}
$$




$$
\text { and, } \begin{aligned}
\exp \left[-\alpha\left(v_{x}^{2}+v_{y}^{2}+v_{\xi}^{2}\right)\right] & \longrightarrow \exp \left[-\alpha\left(v_{x}^{2}+v_{y}^{2}+v_{\xi}^{2}-2 \mathrm{ev} / \mathrm{m}\right)\right] \\
& -\exp \left[-\alpha\left(v^{2}-v_{0}^{2}\right)\right] .
\end{aligned}
$$

Thu term $\sqrt{2 \mathrm{eV} / \mathrm{M}}$ is the modulacing velocity $v_{0}$ as described earlier 1.2.1 and will be a function of time as given by Eq. (1). Both the acceleration process and the evolution of the distribution function are shown schematically in Fig. B-1. In Fig, B-1, the parameter $\alpha$ is the inverse square of the thermal velocity:

$$
a=\frac{M}{2 k T_{I}}=\frac{M}{2 \Delta W_{I}}=\frac{1}{v_{c h}^{2}} .
$$

That this new distribution function is indeed a solution to the Vlasov equation may be verified b; substitution.

It is important to note that the source distribution that emerges is Gausgian ID the veloctty components perpendicular to the normal and approximately exponeatial in the longitudinal compenents.

The normalization constant for this distribution function is writcen in terms of the current density $\mathrm{J}_{0}$ at the source $(\xi=0)$.

$$
\begin{aligned}
J_{0}=n_{0} \bar{v} & =n_{0}\left[A \int \exp \left(-\alpha v_{x}^{2}\right) d v_{x} \int_{j}^{\infty} \exp \left(-\alpha v_{y}^{2}\right) d v_{y} \int_{v_{0}}^{\infty} v_{\xi} \exp -\alpha\left(v_{\xi}^{2}-v_{0}^{2}\right) d v_{\xi}\right] n_{0}^{n_{0}} \\
& =A\left(\frac{\pi}{\alpha}\right) \operatorname{axp}\left(\alpha v_{0}^{2}\right) \int_{v_{0}}^{\infty} v_{\xi} \exp \left(-\alpha v_{\xi}^{2}\right) d v_{\xi}=\frac{A \pi}{2 \alpha^{2}} \\
A & =\frac{2 \alpha^{2} J_{0}}{\pi}
\end{aligned}
$$




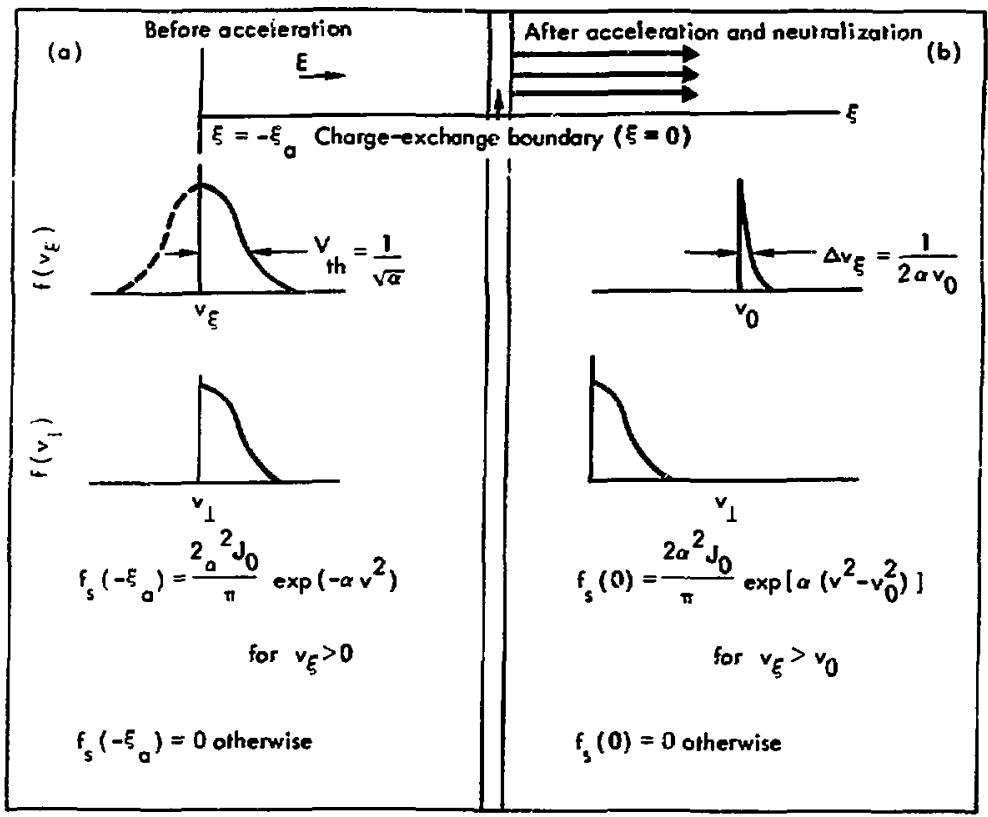

Fig. B1. Acceleration process and evoiution of the distribution function (in real and velocity space), shoiling (a) lons before acceleration and (b) neutrals (charge-exchanged lons) after acceleration. 


\section{Appendix C. Liouville's Theorem}

Liouville's theorem states that, in the neighborhood of a phase point that is following its trafectory, the probability density (or distribution function) remains constant. ${ }^{18}$ The equations of motion determine the trajectory; therefore, given the distribution in phase space at some initial time, the distribution at some later time will be given by rewriting the distribution function in terms of the new coordinates.

$$
f(I, v, t) \equiv f\left(r_{0}, v_{0}, t_{0}\right) \text {. }
$$

In particular, for straight-line trajectorles of neutral particles where the velocity is constant (no interactions or accelerating fields), the equations of motion are

$$
\begin{aligned}
& r=r_{0}+v t, \\
& v=v_{0} .
\end{aligned}
$$

In the problem described in this paper, the distribution function written at some observation point in terms of the distribution function derived at another pcint (the source position). 


\section{Appendix D. Approximate Representation \\ of Distribution Function}

An approximate analytical formula for the distribution function may be derived ${ }^{10}$ by expanding Eqa. (1L) and (13) for $p<+R$ such that

$$
\begin{aligned}
& r \approx p+\rho \mu-\left(1-\mu^{2}\right) \frac{\rho^{2}}{2 R}, \\
& \cos \theta \approx 1-\frac{\left(1-\mu^{2}\right) \rho^{2}}{2 R^{2}} .
\end{aligned}
$$

The value of $v_{\text {min }}[E q .(16)]$ then becomes

$$
v_{\min } \approx \frac{p u t+\left(1-\mu^{3}\right) \frac{p^{2}}{R}}{\left(t_{0}-t\right)} .
$$

Evaluating the modulating velocity [Eq. (7)] at this value of $v$ results in

$$
v_{0}\left(v_{m i n}\right) \approx \frac{-\rho \mu+\left(1-\mu^{2}\right) \frac{\rho^{2}}{R}+\frac{\rho^{3} \mu\left(1-\mu^{2}\right)}{R^{2}}}{\left(t_{0}-t\right)} .
$$

Addit Lonally,

$$
\left.\frac{\partial v_{0}}{\partial v}\right|_{v=v_{m \perp n}} \approx 1+\rho \mu / R-\frac{3\left(1-\mu^{2}\right)}{2 R^{2}} \rho^{2} .
$$

It Is now posstble to express the distribution function in terms of these values. The distribution function is defined as

$$
f \propto \exp \left(-\alpha\left(v^{2}-v_{0}^{2}\right)\right)
$$

After expanding the exponential in a Taylor series about $v_{m i n}$ and substituting In the approximate expressions [Eqs. (D1) to (DS)] 


$$
v^{2}-v_{0}^{2} \approx \frac{\rho^{4} \mu^{2}\left(1-\mu^{2}\right)}{\left(t_{0}-t\right)^{2} R^{2}}+\frac{2 \rho^{2} \mu^{2}\left(v-v_{m i n}\right)}{R\left(t_{0}-t\right)}
$$

the approximate distribution function is then

$f(p, \mu, v, t) \approx \frac{2 \alpha^{2} J_{0}}{\pi} \exp \left(-\alpha\left[\frac{\left(\rho^{4} \mu^{2}\left(1-\mu^{2}\right)\right.}{\left(t_{0}-t\right)^{2} R^{2}}+\frac{2^{\rho^{2} \mu^{2}}\left(v-v_{m i n}\right)}{R\left(t_{0}-t\right)}\right]\right)$.

This expression can be integrated over the speed $v$ to give the approximate expression

$$
F^{\prime}(\rho, \mu, t) \approx \frac{2 \alpha J_{0} R}{\left(t_{0}-t\right)} \exp -\alpha\left[\frac{\rho^{2} \mu^{2}\left(\mu^{2}-1\right)}{R^{2}\left(t_{0}-t\right)^{2}}\right] .
$$




\section{Appendix E. Error Determination For Trapezoidal Integration}

All numerical integrations have an intrinsic error associated with them because the analytical integration is replaced by a sum of terms. Enough of these terms, or grid points, must be used to ensure the destred accuracy of a numerical integration schene.

The code written for this report uses six nested trapezoldal integrations to calculate the lonization prubabilities (F1g. 20). Each integrand must be evaluated at a numer of grid points. It is Important to optimize this number since the total number of calculations required to approximate six Integrations is

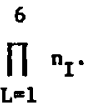

There are two ways to determine that a sufficient imber of grid points have been used for each trapezoldal integration loop. One way is simply to Increase the number of steps unt1l the answers don't change by some predetermined percentage. As an alternate wethod, an equation was derived for determining the number of grid points necessary to ensure a given percentage accuracy. 10

If the integrand $f$ can be approxinated by a quadratic, the percentage error In approximating the Integral with a trapezofdal sum is

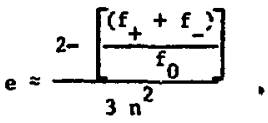

where $f_{+}, f_{0}$, and $f_{-}$are, respectively, the ieginging, middle, and endirg values of the Integrand

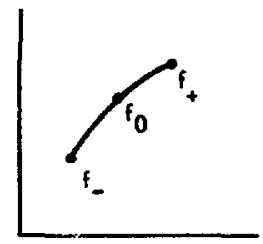


The number of grid points $n$ necessary to ensure a desired percentage accuracy (e.g., for $1 \%, \mathrm{e}=0.01$ ) is

$$
n=\left|\frac{2-\left|\frac{f_{t}+f}{f_{0}}\right|}{0.03}\right| 1 / 2
$$

- If the fntegrand can be approximated by an exponential, exp (ax), then the percentage error incurred by using the trapezofdal integration scheme is

$$
e=\frac{\left[\ln \left(f_{+} / f_{-}\right)\right]^{2}}{12 n^{2}}
$$

\section{Therefore,}

$$
\mathrm{n} \approx \frac{\ln \left(E_{+} / \mathrm{f}_{-}\right)}{2 \sqrt{3 \mathrm{e}}}
$$




\section{Appendix F. Error in the Probability Calculations}

The real increase in ion density due to compression and iontzation is

$$
\frac{d n}{d t}=-n_{I} \nabla \cdot v+n_{N}^{2} \overline{\sigma v}_{N N}+n_{g} n_{I} \overline{\sigma v}_{N I}
$$

Since

$$
\frac{\mathrm{dn}}{\mathrm{dt}}=-\mathbf{n}_{0} \nabla \cdot \mathbf{v}
$$

Eq. (F1) can be written

$$
\frac{d}{d t}\left(\frac{n_{I}}{n_{0}}\right)=\left(\frac{I}{n_{0}}\right) \frac{d n_{I}}{d t}-\left(\frac{n_{I}}{n_{0}^{2}}\right) \frac{d n_{0}}{d t}=\frac{n_{N}}{n_{0}}\left(n_{N} \overline{\sigma v}_{N N}+n_{I} \overline{\sigma v}_{N I}\right) \cdot(F 3)
$$

Therefore, the true probability is overestimated by the fraction $n_{I} / n_{0}$ when the approximation $n_{I} \approx P_{\text {code }} n_{0}$ is used.

$$
\frac{d P_{\text {true }}}{d t}=\left(\frac{n_{N}}{n_{0}}\right) \frac{d P}{d t}=\left(1-\frac{n_{1}}{n_{0}}\right) \frac{d P_{\text {code }}}{d t} .
$$




\section{Appendix G. Derivation of Number Densities from the Continuity Equation for Times Prior to Convergence}

Since the velocity spread of the distribution remains small compared to the streaming velocity for times prior to convergence, the system behaves like a simple cold fluid whth no forces or dissipative effects. Hence, the density at early times can be derived as follows from a macroscopic continuity equation: 10

For mass/charge conservation,

$$
\frac{\partial n}{\partial t}=\frac{1}{\rho^{m}} \frac{\partial\left(\rho^{m} n v\right)}{\partial \rho}=0,
$$

where $m=1$ for cylindrical and $m=2$ for spherical coordinates.

For momentum conservation where the force is zers,

$$
\frac{d v}{d t}=0 \text {, }
$$

where $\frac{d}{d t} \cong \frac{\partial}{\partial t}+v \frac{\partial}{\partial p}$.

ThIs simply states that the velocity of a filuid element at some chosen observation point $(p, t)$ is the same as the value that it had upon emission at the source.

These Eulerian equations can be solved, but the solution can be obtained more directly by transforming to Lagrangian coordinates.

Let the time of emission $t_{e}$ be the Lagranglan variable which identifies any fluid element.

$$
t_{e}=t_{0}-\frac{R}{\rho}\left(t_{0}-t\right)
$$

Inversely,

$$
\rho=R\left(t_{0}-t\right) /\left(t_{0}-t_{e}\right) .
$$

The modulating velocity $v_{0}$ is a function to $t_{e}$ only. From Eq. (7),

$$
v_{0}\left(t_{e}\right)=R /\left(t_{0}-t_{e}\right)
$$


so that Eq. (G1) can be written as

$$
\left(\frac{1}{n}\right) \frac{d n}{d t}=\left(-\frac{1}{\rho^{m}}\right) \frac{\partial\left(\rho^{m} v_{0}\right)}{\partial \rho} \text {. }
$$

which transforms to the Lagrangtan equation

$$
\left(\frac{1}{n}\right) \frac{\partial n}{\partial t}=\frac{m+1}{t_{0}-t} \text {, }
$$

with a solution

$$
n\left(t_{e}, t\right)=\frac{A\left(t_{e}\right)}{\left(t_{0}-t\right)^{m+1}}
$$

The quantity $A\left(t_{e}\right)$ is determined by the boundary condition so that the density as it leaves the source is

$$
n_{0}=\frac{J_{0}}{v\left(t_{e}\right)}
$$

therefore,

$$
n(\rho, t)=\frac{J_{0} R^{m+1}\left(t_{0}-t\right)}{\rho^{m+2}} .
$$

This expression is the same as Eq. (24) where $M=2$ for spherical cooroinates. 


\section{References}

1. R. F. Post, T. K. Fowler, J. Killeen, and A. A. MIrIn, Khys. Rev. Lett. 31,280 (1973).

2. R. F. Post, Lawrence LIvermore Laboratory, private communication.

3. R. F. Post, K. D. Marx, C. J. Eggens, Nucl. Fuaion 15 (1975).

4. K. D. Marx, C. J. (Eggens) Hartman, and R. F. Post, "Approximate Analytlcal Formulas for Systems whlch Employ Convergent Neutral Beams," presented at the Plasma Physics Meeting of the American Physical Society, St. Yetersburg, 1975.

5. W. S. Cooper, K. Halbeck, and S. B. Magyary, "Computer Aided Extractor Design," in Proc. Symp. Ton Sounces and Formation of Beams, ind, BerkeZey, 1974 (Lawrence Berkeley Laboratory, Berkeley, 1974), LBL-3399, Paper II-1.

6. K. A, Berkner, et a1., "Performance of LBL 20-kV, 10-A and 50-A Neutral Beam Infectors," In Proc. Symp. Ion Sources and Formation of Beams, 2nd, Berkeley, 1974 (Lawrence Berkeley Laboratory, Berkeley, 1974), LBL-3399, Paper VI-12.

7. K. W, Ehlers, et al, "Large-Area Plasma Sources," in Proc. Symp. Ion Sources and Formation of Beams, and, Berkeley, 1974 (Lawrence Berkeley Laboratory, Berkeley, 1974), LBL-3399, Paper I-5.

8. C. W. Hartman, Lawrence Livermore Laboratory, private zonmunication.

9. R. N. Sudan, S. Humphrles, and A. Friedman, BuZZ. Am, Phys, Soc. 19, 871 (1974).

10. K. D. Marx, Lawrence LIvermore Laboratory, private communication.

11. C. J. (Eggens) Hartman, K. D. Marx, and R. F. Post, "Numerica1 Calculations of Processes in Convergent Neutral Beams," presented at the Plasma Physics Meeting of the American Physical Soclety, St. Petersourg, 1975.

12. K. D. Marx, A. A. Mirin, M. G. McCoy, M. E. Rensink, and J. Killeen, Caloulation of ( $\sigma \mathrm{v})_{\mathrm{DT}}$ for Anisotropic Mirror and Toroidal Distributions, Lawrence LIvermore Laboratory Rept. UCRL-77873 (1976); submitted to Nuct. Fusion.

13. A. H. Futch, J. P. Holdren, J. Killeen, and A. A. MIrIn, Plasma Phys. 14. 211 (1972). 
14. D. R. Bates and G. W. Griffing, Proc. Phys. Soc. Iond. A66, 961 (1953).

15. J. R, HIskes, Lawrence LIveimore Laboratory, private communication.

16. S. I. Braginski1, Reviewe of Plasma Physics (Consultant's Bureau, New York, 1965).

17. F. F. Chen, Introduction to Plasma Physics (Plenum, New York, 1974).

18. L. Spitzer, Jr., Physics of Fully Ionized Gases, (Intersclence, New York, 1962).

19. J. R. Plerce, Theory and Design of Electron Beams (Van Nostrand, New York, 1954), 2nd ed. 